\title{
Natural Energy Bounds in Quantum Thermodynamics
}

\author{
Daniele Guido $^{1)}$, Roberto Longo ${ }^{2)}$
}

1) Dipartimento di Matematica, Università della Basilicata, I-85100 Potenza, Italy.

2) Dipartimento Matematica, Università di Roma "Tor Vergata", I-00133 Roma, Italy.

\begin{abstract}
Given a stationary state for a noncommutative flow, we study a boundedness condition, depending on a parameter $\beta>0$, which is weaker than the KMS equilibrium condition at inverse temperature $\beta$. This condition is equivalent to a holomorphic property closely related to the one recently considered by Ruelle and D'Antoni-Zsido and shared by a natural class of non-equilibrium steady states. Our holomorphic property is stronger than the Ruelle's one and thus selects a restricted class of non-equilibrium steady states. We also introduce the complete boundedness condition and show this notion to be equivalent to the Pusz-Woronowicz complete passivity property, hence to the KMS condition.

In Quantum Field Theory, the $\beta$-boundedness condition can be interpreted as the property that localized state vectors have energy density levels increasing $\beta$-subexponentially, a property which is similar in the form and weaker in the spirit than the modular compactness-nuclearity condition. In particular, for a Poincaré covariant net of $\mathrm{C}^{*}$-algebras on the Minkowski spacetime, the $\beta$-boundedness property, $\beta \geq 2 \pi$, for the boosts is shown to be equivalent to the Bisognano-Wichmann property. The Hawking temperature is thus minimal for a thermodynamical system in the background of a Rindler black hole within the class of $\beta$-holomorphic states. More generally, concerning the Killing evolution associated with a class of stationary quantum black holes, we characterize KMS thermal equilibrium states at Hawking temperature in terms of the boundedness property and the existence of a translation symmetry on the horizon.
\end{abstract}

Work partially supported by MURST and GNAFA-INDAM 


\section{Introduction.}

In this paper we shall discuss a property for a state which is invariant under a given one-parameter automorphism group of a $\mathrm{C}^{*}$-algebra. This property has two essentially equivalent descriptions either as a boundedness condition or as a holomorphic condition. The boundedness property has a natural interpretation within Quantum Field Theory, being somehow similar to the Haag-Swieca [22] compactness or Buchholz-Wichmann [10] nuclearity-conditions, while the holomorphic condition is a weakening of the KMS thermal equilibrium condition, related to the conditions recently considered by Ruelle [34] and D'Antoni-Zsido [11], thus it naturally pertains to the context of Quantum Statistical Mechanics. It is then natural to discuss our property in a context where both the two above subjects coexist: Black Hole Thermodynamics (see [40]). Our main result is that for a translation invariant Quantum Field Theory such property is equivalent to the KMS condition.

Quantum field theory and the boundedness property. Let us consider a Quantum Field Theory on the Minkowski spacetime and let $\mathfrak{A}(\mathcal{S})$ be the $\mathrm{C}^{*}$-algebra on the vacuum Hilbert space $\mathcal{H}$ generated by the observable localized in the region $\mathcal{S}$. Clearly pure states on the quasi-local observable algebra in the vacuum folium are given by unit vectors of $\mathcal{H}$, unique up to a phase, under the correspondence

$$
\xi \in \mathcal{H},\|\xi\|=1 \longrightarrow \omega_{\xi}
$$

where $\omega_{\xi}$ is the expectation functional $\omega_{\xi}(X) \equiv(X \xi, \xi)$. Denote by $\mathcal{L}(\mathcal{S})$ the set of vector states localized in $\mathcal{S}$, namely

$$
\mathcal{L}(\mathcal{S}) \equiv\left\{\xi \in \mathcal{H},\|\xi\|=1:\left.\omega_{\xi}\right|_{\mathfrak{A}\left(\mathcal{S}^{\prime}\right)}=\left.\omega_{0}\right|_{\mathfrak{A}\left(\mathcal{S}^{\prime}\right)}\right\},
$$

with $\omega_{0}=\omega_{\Omega}$ the vacuum state.

It easy to see that $\xi \in \mathcal{L}(\mathcal{S})$ if and only if there exists an isometry $W \in$ $\mathfrak{A}\left(\mathcal{S}^{\prime}\right)^{\prime}$ such that $\xi=W \Omega$; indeed $W$ is the closure of the map $X^{\prime} \Omega \rightarrow X^{\prime} \xi$, $X^{\prime} \in \mathfrak{A}\left(\mathcal{S}^{\prime}\right)$. Therefore, if Haag duality holds for $\mathcal{S}$, namely $\mathcal{A}(\mathcal{S})=\mathfrak{A}\left(\mathcal{S}^{\prime}\right)^{\prime}$, where $\mathcal{A}(\mathcal{S})=\mathfrak{A}(\mathcal{S})^{\prime \prime}$ is the weak closure of $\mathfrak{A}(\mathcal{S})$, we have

$$
\mathcal{L}(\mathcal{S})=\left\{W \Omega: W \in \mathcal{A}(\mathcal{S}), W^{*} W=1\right\} .
$$

The $\beta$-boundedness condition demands that all vectors in $\mathcal{L}(\mathcal{S})$ have energy density levels increasing $\beta$-subexponentially, namely

$$
\int e^{-\lambda \beta} \mathrm{d} \mu_{\xi}(\lambda)<+\infty, \forall \xi \in \mathcal{L}(\mathcal{S}),
$$

where $\mu_{\xi}(B)=\int_{B} \mathrm{~d}(E(\lambda) \xi, \xi)$ is the spectral measure associated by the Hamiltonian $H$ to $\xi, H=\int \lambda \mathrm{d} E(\lambda)$ being the spectral resolution of $H$. By the spectral theorem, equation $(\overline{0.2})$ is equivalent to $\mathcal{L}(\mathcal{S}) \subset D\left(e^{-\frac{\beta}{2} H}\right)$.

Since every operator $X \in \mathfrak{A}(\mathcal{S})$ with $\|X\|<1$ is a convex combination of unitaries in $\mathfrak{A}(\mathcal{S})$ (see e.g [29]), the $\beta$-boundedness condition is equivalent to 
$\mathfrak{A}(\mathcal{S}) \Omega \subset D\left(e^{-\frac{\beta}{2} H}\right)$ and turns out to be equivalent to

$$
e^{-\beta H} \mathfrak{B}_{1} \Omega \text { is a bounded set }
$$

for some, hence for all, weakly dense ${ }^{*}$-subalgebra $\mathfrak{B}$ of $\mathcal{A}(\mathcal{S})$, where $\mathfrak{B}_{1}$ denotes the unit ball of $\mathfrak{B}$.

Equation (0.2) already shows an interesting aspect of the $\beta$-boundedness property in Quantum Field Theory, namely that, as mentioned, it can be formulated much in analogy with the compactness-nuclearity [22, 10].

Now, we do not have yet specified which region $\mathcal{S}$ is supposed to be. It could be the entire Minkowski spacetime $M$, but an unbounded region as a spacelike cone will already contain the relevant information. As $H$ commutes with all translations, property $(0.3)$ for $\mathcal{S}$ do implies the same property for the translated regions $\left\{\mathcal{S}+x, x \in \mathbb{R}^{d+1}\right\}$, hence for the quasi-local algebra. It is then almost immediate that the $\beta$-boundedness condition is equivalent to $H$ to be semibounded, thus, by Poincaré covariance, to the positivity of the energymomentum.

Although instructive, the boundedness condition would certainly have a limited interest if were confined to the above situation. Such condition acquires however a deeper role when the Hamiltonian is not positive, as is the case in thermodynamical contexts. To better illustrate this point, we need to make a digression in Statistical Mechanics and discuss a property equivalent to the boundedness condition.

$\beta$-holomorphy and non-equilibrium states. As is well known, the thermal equilibrium states in Quantum Statistical Mechanics at finite volume are the Gibbs states, while at infinite volume they are KMS [21] states, the proper generalization of the former, which are defined as follows.

Let $\mathfrak{A}$ be a $\mathrm{C}^{*}$-algebra and $\alpha$ a one-parameter group of automorphisms of $\mathfrak{A}$ and $\mathfrak{B}$ a dense *-subalgebra of $\mathfrak{A}$. A state $\omega$ of $\mathfrak{A}$, namely a normalized positive linear functional of $\mathfrak{A}$, is a KMS state for $\alpha$ at inverse temperature $\beta>0$ if, for any $X, Y \in \mathfrak{B}$, the function

(a) $F_{X, Y}(t) \equiv \omega\left(\alpha_{t}(X) Y\right)$ extends to a function in $A\left(S_{\beta}\right)$,

(b) $F_{X, Y}(t+i \beta)=\omega\left(Y \alpha_{t}(X)\right)$,

where $A\left(S_{\beta}\right)$ is the algebra of functions analytic in the strip $S_{\beta}=\{0<\Im z<\beta\}$, bounded and continuous on the closure $\bar{S}_{\beta}$. The consideration of the subalgebra $\mathfrak{B}$ is indeed unnecessary (but convenient for future reference) as properties $(a)$ and $(b)$ then hold for all $X, Y \in \mathfrak{A}$.

Let us now consider non-equilibrium statistical mechanics and in particular the physical situation recently considered by Ruelle [34. There a quantum system $\Sigma$ is interacting with a set of infinite reservoirs $R_{k}$ that are in equilibrium at different temperatures $\beta_{k}^{-1}$. The system $\Sigma$ may be acted upon by a force, that we assume to be time independent. In this context, at least if $\Sigma$ is finite, a natural class of stationary non-equilibrium states occur, the non-equilibrium steady states. If we denote as above the observable $\mathrm{C}^{*}$-algebra by $\mathfrak{A}$ and the 
time evolution automorphism group by $\alpha$, a non-equilibrium steady state $\omega$ of $\mathfrak{A}$ satisfies property $(a)$ in the KMS condition, for all $X, Y$ in a dense ${ }^{*}$-subalgebra of $\mathfrak{B}$, with $\beta=\min \beta_{k}$, but not necessarily property $(b)$.

States with property $(a)$ have been independently also discussed by D'Antoni and Zsido [11]. A typical example of a non-KMS state satisfying property $(a)$ is provided by the tensor product of KMS states at different temperatures; in this case the parameter $\beta$ is clearly the minimum of the inverse temperatures. Further examples are obtained by considering a KMS state with respect to a bounded perturbation of the dynamics, showing a certain stability of the holomorphic property. In these examples the states satisfy property $(a)$.

Because of property $(b)$, KMS states also satisfy the bound

(c) $|F(t+i \beta)| \leq C\|X\|\|Y\|$ for some constant $C>0$.

$X, Y \in \mathfrak{B}$, indeed one may take $C=1$. We shall show that the bound $(c)$ automatically occurs if property $(a)$ holds true for all the elements of a $\mathrm{C}^{*}$ algebra $\mathfrak{A}$.

We say that a $\alpha$-invariant state $\omega$ is $\beta$-holomorphic if Properties $(a)$ and (c) hold for all $X, Y$ in a dense ${ }^{*}$-subalgebra $\mathfrak{B}$ or, equivalently, if Property $(a)$ holds for all $X, Y$ in the $\mathrm{C}^{*}$-algebra $\mathfrak{A}$.

Since the bound $(c)$ does not necessarily hold for the Non-Equilibrium Steady States considered by Ruelle [34], $\beta$-holomorphic states form a subclass of the class of such non-equilibrium states that, in a sense, are closer to equilibrium.

$K M S$ and complete $\beta$-boundedness. Because of the bound $(c)$, the $\beta$-holomorphic property for a state $\omega$ turns out to be equivalent to the $\frac{\beta}{2}$-boundedness property for the Hamiltonian $H$ in the GNS representation. The two properties are thus two different aspects of the same notion.

Furthermore, if the constant $C$ is equal to 1 , we may use an inequality of Pisier 31, improved by Haagerup 24, to get the inequality

$$
e^{\mp \beta H} \leq 1+\Delta^{ \pm 1}
$$

where $\Delta$ is the modular operator associated with the GNS vector $\Omega$ that, for simplicity, we are assuming to be separating for the weak closure $\pi_{\omega}(\mathfrak{A})^{\prime \prime}$. Thus the $\beta$-holomorphic property with $C=1$ entails that the Hamiltonian $H$ is dominated, in the above sense, by the thermal equilibrium Hamiltonian $\log \Delta$.

A better understanding of the $\beta$-holomorphic property is then obtained by comparing it with the passivity condition of Pusz-Woronowicz [32], which is an expression of the second principle of thermodynamics. As is known, the passivity condition is weaker than the KMS condition, while the complete passivity turns out to be equivalent to the KMS property at some inverse temperature (possibly 0 or $+\infty$ ). In analogy, we define the complete $\beta$-holomorphic property and show this property to be equivalent to complete passivity, thus to the KMS condition.

We now return to Quantum Field Theory in a more general context.

Black hole thermodynamics: minimality of the Hawking temperature. During the past thirty years, a theory of black hole thermodynamics has been developed much in analogy with classical thermodynamics, see 40]. In this new context 
the thermodynamical functions acquire a new meaning, for example the entropy is proportional to the area of the black hole [2, yet a "generalized second law of thermodynamics" holds.

As derived by Hawking [24], the temperature appearing in this formula is a true physical temperature, in other words black holes do emit a thermal radiation, provided quantum effects are taken into accounts.

This effect, or its closely related Unruh effect in the Rindler spacetime [39], has been noticed [36 to be essentially equivalent to the Bisognano-Wichmann property in Quantum Field Theory.

Let now a Quantum Field Theory on the Minkowski spacetime $M$ be specified by the algebras $\mathfrak{A}(\mathcal{O})$ of the observables localized in the regions $\mathcal{O}$. As is known the Rindler spacetime can be identified with a wedge region $W$ of $M$, say $W=\left\{x \in \mathbb{R}^{d+1}: x_{1}>\left|x_{0}\right|\right\}$. Thus $W$ represents the exterior of a Rindler black hole. The pure Lorentz transformation in the $x_{1}$-direction on $M$ leave $W$ globally invariant and thus give rise to a one-parameter automorphism group $\alpha$ of the $\mathrm{C}^{*}$-algebra $\mathfrak{A}(W)$.

The Bisognano-Wichmann theorem shows that, if $\mathfrak{A}$ is generated by a Wightman field, then the restriction to $\mathfrak{A}(W)$ of the vacuum state satisfies the KMS condition with respect to $\alpha$ at inverse temperature $\beta=2 \pi$. One can then explain the Unruh effect on this basis, see 36.

We shall show that the $\beta$-boundedness property, $\beta \geq 2 \pi$, in the above Quantum Field Theory context, actually implies the KMS property at $\beta=2 \pi$, namely the state is at thermal equilibrium.

In particular, for a Poincaré covariant net of local observable algebras on the Minkowski spacetime, we obtain a characterization of the Bisognano-Wichmann property: there should exist a spacelike cone $\mathcal{S}$ contained in the wedge $W$ and a weakly dense *-algebra $\mathfrak{B}$ of $\mathcal{A}(\mathcal{S})=\mathfrak{A}(\mathcal{S})^{\prime \prime}$ such that

$$
e^{-\pi K} \mathfrak{B}_{1} \Omega \text { is a bounded set }
$$

of the underlying Hilbert space. Here $\Omega$ is the vacuum vector and $K$ is the generator of the boost unitary group corresponding to $W$, namely the Killing Hamiltonian for the Rindler space $W$. Note that, if the Bisognano-Wichmann property and the split property [12] hold, then also the modular compactness condition holds true, namely $e^{-\overline{\lambda K}} \mathfrak{B}_{1} \Omega$ is a compact set for all $0<\lambda<\pi$ [8] $\left(e^{-2 \pi K}\right.$ is then the modular operator associated with $\left.(\mathcal{A}(W), \Omega)\right)$. Thus the boundedness condition (0.4) is very similar in the form to the modular compactness-nuclearity condition 8 , 9].

Stating our result in the setting of Rindler spacetime, we obtain the following: if a state $\omega$ is $\beta$-holomorphic, with respect to the Killing evolution, with parameter $\beta^{-1}$ less or equal to the Hawking temperature, then $\omega$ is indeed a KMS state at Hawking temperature.

This minimality character of the Hawking temperature may have a further physical interpretation, which is however limited by the fact that only $\beta$-holomorphic states appear in the context, see the conclusion at the end of this paper. 
We then extend our analysis to stationary stationary black holes described by a globally hyperbolic spacetime with bifurcate Killing horizon, making use of the net of observables localized on the horizon, as in [19, 28]. We show that, assuming the $\beta$-boundedness, the KMS condition is equivalent to the existence of a translation symmetry on the horizon.

Our paper is organized in two sections. In the first one we deal with $\mathrm{C}^{*}$ algebras and automorphisms or endomorphisms; we discuss there the basic structure provided by the $\beta$-holomorphic and $\beta$-boundedness condition, that we later apply in Quantum Field Theory in Section 2. In this second section we first discuss our results in the Minkowski, or Rindler, spacetime; then we study the corresponding structure for one-dimensional nets and we then apply these results to the case of a Quantum Field Theory on a spacetime with bifurcate Killing horizon.

\section{Holomorphic states and the boundedness prop- erty}

In this section we discuss the basic structure of the holomorphic and of the boundedness properties. In the next section we shall apply our results to the Quantum Field Theory context.

\subsection{General properties}

Let $\mathcal{H}$ be a Hilbert space, $\Omega \in \mathcal{H}$ a vector and $K$ a selfadjoint operator on $\mathcal{H}$.

We shall say that a linear subspace $\mathfrak{S}$ of $B(\mathcal{H})$ is $\beta$-bounded with respect to $K$ and $\Omega$ if $\mathfrak{S} \Omega \subset D\left(e^{-\beta K}\right)$, the domain of $e^{-\beta K}$, and the linear map

$$
\Phi_{\beta}: X \in \mathfrak{S} \rightarrow e^{-\beta K} X \Omega \in \mathcal{H}
$$

is bounded.

Note that if $\mathfrak{S}$ is $\beta$-bounded then it is $\beta^{\prime}$-bounded for all $0<\beta^{\prime} \leq \beta$, indeed if $E$ is the spectral projection of $K$ relative to the interval $[0,+\infty)$, then

$$
\begin{aligned}
& \left\|e^{-\beta^{\prime} K} X \Omega\right\|^{2}=\left\|E e^{-\beta^{\prime} K} X \Omega\right\|^{2}+\left\|(1-E) e^{-\beta^{\prime} K} X \Omega\right\|^{2} \\
\leq & \|X \Omega\|^{2}+\left\|(1-E) e^{-\beta K} X \Omega\right\|^{2} \leq\|X\|^{2}+\left\|e^{-\beta K} X \Omega\right\|^{2} \leq 1+\left\|\Phi_{\beta}\right\|^{2},
\end{aligned}
$$

for all $X \in \mathfrak{S}_{1}$, where $\mathfrak{S}_{1}$ denotes the unit ball of $\mathfrak{S}$.

Lemma 1.1. Let $\mathfrak{S}$ be a $\beta$-bounded linear subspace of $B(\mathcal{H})$ for some $\beta>0$. Then the restriction of $\Phi=\Phi_{\beta}$ to $\mathfrak{S}_{1}$ is continuous with the weak-operator topology on $\mathfrak{S}$ and the weak topology on $\mathcal{H}$.

If $\mathfrak{S}$ is norm closed and $\mathfrak{S} \Omega$ is contained in $D\left(e^{-\beta K}\right)$, then $\mathfrak{S}$ is automatically $\beta$-bounded. 
Proof. Assume then that $\Phi$ is bounded. To check the continuity, let $X_{i} \in \mathfrak{S}_{1}$ be a net weakly convergent to 0 . Then for all $\xi \in D\left(e^{-\beta K}\right)$ we have

$$
\lim _{i}\left(\xi, \Phi\left(X_{i}\right)\right)=\lim _{i}\left(\xi, e^{-\beta K} X_{i} \Omega\right)=\lim _{i}\left(e^{-\beta K} \xi, X_{i} \Omega\right)=0 .
$$

as $D\left(e^{-\beta K}\right)$ is dense in $\mathcal{H}$ and $\left\{\Phi\left(X_{i}\right)\right\}_{i}$ is bounded by assumption, it follows that $\Phi\left(X_{i}\right) \rightarrow 0$ weakly.

It remains to show that $\Phi$ is bounded if $\mathfrak{S}$ is norm closed. We shall show that $\Phi$ is closable, thus $\Phi$ will be bounded by the closed graph theorem.

Let $\left\{X_{n}\right\}$ be a sequence in $\mathfrak{S}$ and $\eta \in \mathcal{H}$ be such that $X_{n} \rightarrow 0$ and $\Phi\left(X_{n}\right) \rightarrow \eta$ in norm. As $\left\{X_{n}\right\}$ is a bounded set, by the just proved weak continuity of $\Phi$ we have that $\Phi\left(X_{n}\right) \rightarrow 0$ weakly, thus $\eta=0$.

Lemma 1.2. Let $\mathcal{M}$ be a von Neumann algebra on the Hilbert space $\mathcal{H}, \Omega$ a vector, $K$ a selfadjoint operator of $\mathcal{H}$ and $\beta>0$.

The following are equivalent:

(i) There exists a weakly dense ${ }^{*}$-subalgebra $\mathfrak{B}$ of $\mathcal{M}$ which is $\beta$-bounded (w.r.t. $K$ and $\Omega$ );

(ii) $\mathcal{M}$ is $\beta$-bounded;

(iii) $\mathfrak{A} \Omega \subset D\left(e^{-\beta K}\right)$, where $\mathfrak{A}$ is some weakly dense $C^{*}$-subalgebra of $\mathcal{M}$.

In this case $\left.\Phi_{\beta}\right|_{\mathfrak{B}}$ and $\left.\Phi_{\beta}\right|_{\mathcal{M}}$ have the same norm.

Proof. Assuming in $(i)$ that $e^{-\beta K} \mathfrak{B}_{1} \Omega$ is contained in the ball of radius $C>0$, we shall show that the same is true for $e^{-\beta K} \mathcal{M}_{1} \Omega$, i.e. (ii) holds. Let $X \in \mathfrak{B}$. By Kaplanski density theorem [38], there exists a net of operators $X_{i} \in \mathfrak{B}_{1}$ strongly convergent to $X$. Since $\left\|e^{-\beta K} X_{i} \Omega\right\| \leq C$, we may assume, possibly restricting to a subnet, that $e^{-\beta K} X_{i} \Omega$ weakly converges to $\eta \in \mathcal{H},\|\eta\| \leq C$. Now take $\xi \in \mathcal{D}\left(e^{-\beta K}\right)$. We have

$$
(\xi, \eta)=\lim _{i}\left(\xi, e^{-\beta K} X_{i} \Omega\right)=\lim _{i}\left(e^{-\beta K} \xi, X_{i} \Omega\right)=\left(e^{-\beta K} \xi, X \Omega\right) .
$$

Since $e^{-\beta K}$ is self-adjoint, this means that $X \Omega \in \mathcal{D}\left(e^{-\beta K}\right)$ and $e^{-\beta K} X \Omega=\eta$, i.e. $\left\|e^{-\beta K} \mathcal{M}_{1} \Omega\right\| \leq C$.

Now $(i i) \Rightarrow($ iii $)$ and $(i i i) \Rightarrow(i)$ follows by Lemma 1.1 as $\mathfrak{A}$ is norm closed.

Let now $\mathfrak{A}$ be a unital $\mathrm{C}^{*}$-algebra, $\alpha$ a one-parameter automorphism group of $\mathfrak{A}$ and $\omega$ a $\alpha$-invariant state of $\mathfrak{A}$. We shall always assume that the maps $t \in \mathbb{R} \rightarrow \omega\left(\alpha_{t}(X) Y\right)$ are continuous for all $X, Y \in \mathfrak{A}$. Denote by $(\mathcal{H}, \pi, \Omega)$ the GNS triple associated with $\omega$ and by $U$ the one-parameter unitary group on $\mathcal{H}$ implementing $\pi \cdot \alpha$ :

$$
U(t) \pi(X) \Omega=\pi\left(\alpha_{t}(X)\right) \Omega, \quad X \in \mathfrak{A} .
$$

¿From our continuity assumption, it follows at once that $U$ is strongly continuous. Denote by $K$ the infinitesimal generator of $U$. 
Proposition 1.3. If $\omega$ is a pure state, $\pi(\mathfrak{A})$ is $\beta$-bounded with respect to $K$ and $\Omega$ if and only if the spectrum of $K$ is bounded below.

Proof. If $\omega$ is pure, then $\mathcal{M} \equiv \pi(\mathfrak{A})^{\prime \prime}=B(\mathcal{H})$, hence, if the boundedness condition holds, $e^{-\beta K} B(\mathcal{H})_{1} \Omega$ is bounded. As $B(\mathcal{H})_{1} \Omega=\mathcal{H}_{1}$, it follows that $e^{-\beta K}$ is bounded, thus $K$ is semibounded. The converse is obvious.

Let $\mathfrak{B}$ a ${ }^{*}$-subalgebra of $\mathfrak{A}$. The state $\omega$ of $\mathfrak{A}$ is $\beta$-holomorphic on $\mathfrak{B}$ if $\omega$ is $\alpha$-invariant and for every $X, Y \in \mathfrak{B}$ the function $F_{X, Y}(t)=\omega\left(\alpha_{t}(X) Y\right)$ is the boundary value of a function in holomorphic in the strip $S_{\beta}=\{z: 0<\Im z<\beta\}$, continuous in $\bar{S}_{\beta}$.

Denote by $A\left(S_{\beta}\right)$ the algebra of functions holomorphic in $S_{\beta}$, bounded and continuous in its closure $\bar{S}_{\beta}$.

Proposition 1.4. Let $\mathfrak{A}, \alpha, \omega, K, \Omega$ as before, $\mathfrak{B} a^{*}$-subalgebra of $\mathfrak{A}$. Then $\omega$ is $\beta$-holomorphic on $\mathfrak{B}$ if and only if $\pi(\mathfrak{B}) \Omega \subset D\left(e^{-\frac{\beta}{2} K}\right)$.

In this case $F_{X, Y}$ extends to a function in $A\left(S_{\beta}\right)$, for all $X, Y \in \mathfrak{B}$.

Proof. Immediate by Lemma 1.17.

Theorem 1.5. Let $\mathfrak{A}, \alpha, \omega, K, \Omega$ as before, $\mathfrak{B} a^{*}$-subalgebra of $\mathfrak{A}$. Then $\pi(\mathfrak{B})$ is $\frac{\beta}{2}$-bounded w.r.t. $K$ and $\Omega$ if and only if $\omega$ is $\beta$-holomorphic on $\mathfrak{B}$ and

$$
\left|F_{X, Y}(t+i \beta)\right| \leq C\|X\|\|Y\|,
$$

for some constant $C>0$.

If moreover $\mathfrak{B}$ is norm closed, then $\pi(\mathfrak{B})$ is $\beta / 2$-bounded w.r.t. $K$ and $\Omega$ if and only if $\omega$ is $\beta$-holomorphic for $\mathfrak{B}$.

Proof. Clearly $F_{Y^{*}, X}(t)=\left(e^{i t K} \pi(X) \Omega, \pi(Y) \Omega\right)$ for all $X, Y \in \mathfrak{A}$. If $\omega$ is $\beta$ holomorphic on $\mathfrak{B}$ and $X, Y \in \mathfrak{B}$, then $F_{Y^{*}, X}$ is boundary value of a function in $A\left(S_{\beta}\right)$, thus by Lemma $1.17 \pi(\mathfrak{B}) \Omega \subset D\left(e^{-\frac{\beta}{2} K}\right)$. If moreover the bound (1.2) holds, then

$\left\|\Phi_{\beta / 2}||_{\mathfrak{B}}\right\|^{2}=\sup _{X, Y \in \mathfrak{B}_{1}}\left|\left(e^{-\frac{\beta}{2} K} \pi(X) \Omega, e^{-\frac{\beta}{2} K} \pi(Y) \Omega\right)\right|=\sup _{X, Y \in \mathfrak{B}_{1}}\left|F_{Y^{*}, X}(i \beta)\right| \leq C$

thus $\mathfrak{B}$ is $\beta / 2$-bounded.

Conversely, if $\mathfrak{B}$ is $\beta / 2$-bounded, then $\pi(\mathfrak{B}) \Omega \subset D\left(e^{-\frac{\beta}{2} K}\right)$ and the same computation done above yields, by Lemma 1.17, that $F_{X, Y} \in A\left(S_{\beta}\right)$ for all $X, Y \in \mathfrak{B}$ and

$$
\sup _{X, Y \in \mathfrak{B}_{1}}\left|F_{X, Y}(t+i \beta)\right|=\sup _{X, Y \in \mathfrak{B}_{1}}\left|F_{X, Y}(i \beta)\right|=\left\|\left.\Phi_{\beta / 2}\right|_{\mathfrak{B}}\right\|^{2} .
$$

so the the bound (1.2) holds with $C=\left\|\left.\Phi_{\beta / 2}\right|_{\mathfrak{B}}\right\|^{2}$.

Since by Prop. 1.4 $\omega$ is $\beta$-holomorphic on $\mathfrak{B}$ iff $\pi(\mathfrak{B}) \Omega \subset D\left(e^{-\frac{\beta}{2} K}\right)$, the rest follows by Lemma 1.2 . 
Of course KMS states at inverse temperature $\beta^{\prime}>0$ are $\beta$-holomorphic for all $0<\beta \leq \beta^{\prime}$ on all $\mathfrak{A}$ and satisfy the bound (1.2).

Corollary 1.6. With the above notations, let $\omega$ be $\beta$-holomorphic on $\mathfrak{B}$. Then $\omega$ is $\beta$-holomorphic on the closure $\overline{\mathfrak{B}}$ iff the bound (1.2) holds.

Proof. Immediate by Lemma 1.2 and Theorem 1.5 .

\subsection{Complete $\beta$-holomorphy and KMS condition}

We begin to recall the following inequality $(1.3)$ which is due to Pisier, with the improved constant due to Haagerup, see [30].

Theorem 1.7. 31, 23]. Let $\Phi$ be a bounded linear map from a $C^{*}$-algebra $\mathfrak{A}$ to a Hilbert space $\mathcal{H}$. Then there exist two states $\varphi$ and $\psi$ on $\mathfrak{A}$ such that

$$
\|\Phi(X)\|^{2} \leq\|\Phi\|^{2}\left(\varphi\left(X^{*} X\right)+\psi\left(X X^{*}\right)\right), \quad X \in \mathfrak{A} .
$$

In the special case where $\mathfrak{A}$ is unital and $\|\Phi\|=\|\Phi(1)\|$, one may take $\varphi=\psi=$ $\|\Phi\|^{-1}(\Phi(\cdot), \Phi(1))$ in eq. (1.3).

The special case in last part of the statement is obtained during Haagerup's proof of the inequality (1.3); such proof is not difficult and can be found in [30], Thm 7.3.

Corollary 1.8. Let $\mathcal{M}$ be a von Neumann algebra on a Hilbert space $\mathcal{H}, \Omega \in \mathcal{H}$ a cyclic unit vector for $\mathcal{M}$ and $U(t)=e^{i t K}$ a $\Omega$-fixing one-parameter unitary group on $\mathcal{H}$ implementing automorphisms of $\mathcal{M}$.

If $\left\|e^{-\beta K} \mathcal{M}_{1} \Omega\right\| \leq 1$, then

$$
e^{-2 \beta K} \leq 1+\Delta E,
$$

where $E \in \mathcal{M}$ is the projection onto $\mathcal{H}_{0} \equiv \overline{\mathcal{M}^{\prime} \Omega}$ and $\Delta$ is the modular operator on $\mathcal{H}_{0}$ associated with $(E \mathcal{M} E, \Omega)$.

Proof. As the map $\Phi_{\beta}: X \in \mathcal{M} \rightarrow e^{-\beta K} X \Omega \in \mathcal{H}$ satisfies $\left\|\Phi_{\beta}\right\|=\left\|\Phi_{\beta}(1)\right\|=$ 1 , the inequality (1.3) holds with $\varphi=\psi=\omega$ (see 1.7), where

$$
\omega(X)=\left(\Phi_{\beta}(X), \Phi_{\beta}(1)\right)=(X \Omega, \Omega)
$$

namely

$$
\left\|e^{-\beta K} X \Omega\right\|^{2} \leq\|X \Omega\|^{2}+\left\|X^{*} \Omega\right\|^{2}, X \in \mathcal{M} .
$$

Assuming first that $\Omega$ is also separating, i.e. $E=1$, if $X \in \mathcal{M}$ and $X \Omega \in D(\Delta)$ we then have

$$
\left(e^{-\beta K} X \Omega, e^{-\beta K} X \Omega\right) \leq(X \Omega, X \Omega)+(\Delta X \Omega, X \Omega)=((1+\Delta) X \Omega, X \Omega) .
$$

Since $U$ implements automorphims of $\mathcal{M}$ and $U(t) \Omega=\Omega$, by the modular theory $U(t)$ and $\Delta^{i s}$ commute, thus there exists a strongly dense subalgebra $\mathfrak{B}$ of $\mathcal{M}$ 
such that $\mathfrak{B} \Omega$ is a core for every continuous function of $K$ or of $\log \Delta$. Taking $X \in \mathfrak{B}$, the above equation gives

$$
\left(e^{-2 \beta K} X \Omega, X \Omega\right) \leq((1+\Delta) X \Omega, X \Omega),
$$

thus $e^{-2 \beta K} \leq 1+\Delta$ as $\mathfrak{B} \Omega$ is a core for both $e^{-2 \beta K}$ and $1+\Delta$.

In general, if $E \neq 1$, we may consider the the reduced von Neumann algebra $E \mathcal{M} E$ on $\mathcal{H}_{0}$. Since $\Omega$ is separating for $E \mathcal{M} E$ and $K$ commutes with $E$, the above shows that

$$
e^{-2 \beta K} E \leq E+\Delta E .
$$

Thus, by the eq. 1.5), we have for all $X \in \mathcal{M}$

$$
\left\|e^{-\beta K}(1-E) X \Omega\right\|^{2} \leq\|(1-E) X \Omega\|^{2}+\left\|X^{*}(1-E) \Omega\right\|^{2}=\|(1-E) X \Omega\|^{2},
$$

that entails

$$
e^{-2 \beta K}(1-E) \leq 1-E .
$$

Combining the inequalities (1.6) and (1.7) we get the desired inequality (1.4).

Remark 1.9. In general, the tensor product of bounded maps from $\mathrm{C}^{*}$-algebras to Hilbert spaces is not bounded. Indeed, if $\mathcal{M}$ is a von Neumann algebra with a cyclic and separating vector $\Omega$, the map $\Phi_{\alpha}: X \in \mathcal{M} \mapsto \Delta^{\alpha} X \Omega$ is bounded for any $\alpha \in[0,1 / 2]$, but $\Phi_{\alpha} \otimes \Phi_{\beta}$ is not necessarily bounded, if $\alpha \neq \beta$.

As an example, let $\mathcal{M} \simeq B(\mathcal{H})$ be a type $\mathrm{I}_{\infty}$ factor acting by right multiplication on the Hilbert space $L^{2}(\mathcal{H})$ of the Hilbert-Schmidt operators affiliated on $\mathcal{H}, h$ a positive Hilbert-Schmidt operator of norm 1 in $L^{2}(\mathcal{H})$. Then $\Delta X=h^{2} X h^{-2}, X \in L^{2}(M)$, and $\Phi_{\alpha}(X)=h^{2 \alpha} X h^{1-2 \alpha}, X \in \mathcal{M}, X \in L^{2}(\mathcal{H})$. As a consequence, if $h^{2 \alpha}$ and $h^{1-2 \alpha}$ are both are Hilbert-Schmidt, the norm of the map $\Phi_{\alpha}$ is bounded by $\left\|h^{2 \alpha}\right\|_{2} \cdot\left\|h^{1-2 \alpha}\right\|_{2}$. Therefore, $\left\|\Phi_{\alpha} \otimes \Phi_{\beta}\right\| \leq$ $\left\|h^{2 \alpha}\right\|_{2} \cdot\left\|h^{1-2 \alpha}\right\|_{2} \cdot\left\|h^{2 \beta}\right\|_{2} \cdot\left\|h^{1-2 \beta}\right\|_{2}$. For example, if the eigenvalue sequence of $h$ is $\left\{2^{-n}\right\}_{n \in \mathbb{N}}, \Phi_{\alpha} \otimes \Phi_{\beta}$ is bounded for every $\alpha, \beta \in(0,1 / 2)$.

On the other hand, denoting by $F$ the unitary operator in $\mathcal{M} \otimes \mathcal{M}$ that, under the isomorphism with $B(\mathcal{H} \otimes \mathcal{H})$, is defined by $F \xi \otimes \eta=\eta \otimes \xi$, one has $F(X \otimes Y)=(Y \otimes X) F$, hence

$$
\Phi_{\alpha} \otimes \Phi_{\beta}(F)=h^{2 \alpha} \otimes h^{2 \beta} F h^{1-2 \alpha} \otimes h^{1-2 \beta}=h^{1-\varepsilon} \otimes h^{1+\varepsilon} F
$$

where $\varepsilon=2 \alpha-2 \beta$. Thus, if $h^{1-\varepsilon}$ or $h^{1+\varepsilon}$ are not Hilbert-Schmidt, $\Phi_{\alpha} \otimes \Phi_{\beta}$ is unbounded. For example, if the eigenvalue sequence of $h$ is $\left\{\frac{1}{\sqrt{n} \log n}\right\}_{n \in \mathbb{N}}$, then $\Phi_{\alpha} \otimes \Phi_{\beta}$ is bounded if and only if $\alpha=\beta$.

Note that, if in Corollary 1.8 the vector $\Omega$ is separating for $\mathcal{M}$, then by modular theory $J \Delta J=\Delta^{-1}$ and $J K J=-K$, where $J$ is the modular conjugation of $(\mathcal{M}, \Omega)$, thus the inequality $e^{2 \beta K} \leq 1+\Delta^{-1}$ holds too, therefore

$$
e^{\mp 2 \beta K} \leq 1+\Delta^{ \pm 1}
$$


showing that the $\beta$-boundedness condition with constant $C=1$ sets a a bound on the Hamiltonian $K$ by the equilibrium Hamiltonian $\log \Delta$.

It is then natural to look for further conditions that entail $K$ to be proportional to $\log \Delta$.

Let $\mathfrak{A}$ be a $\mathrm{C}^{*}$-algebra acting on a Hilbert space $\mathcal{H}$ with a cyclic vector $\Omega \in \mathcal{H}$ and $U(t)=e^{i t K}$ a $\Omega$-fixing one-parameter unitary group on $\mathcal{H}$ implementing automorphisms of $\mathfrak{A}$. We shall say that $\mathfrak{A}$ is completely $\beta$-bounded with respect to $K$ and $\Omega$ if $\left\|\Phi_{\beta} \otimes \Phi_{\beta} \otimes \cdots \otimes \Phi_{\beta}\right\| \leq 1$, for all finitely many tensor products of $\Phi_{\beta}$ with itself, with $\Phi_{\beta}: X \in \mathfrak{A} \rightarrow e^{-\beta K} X \Omega \in \mathcal{H}$.

Here we consider the spatial tensor product norm on $\mathfrak{A} \otimes \mathfrak{A} \otimes \cdots \otimes \mathfrak{A}$ and, of course, the Hilbert space tensor norm on $\mathcal{H} \otimes \mathcal{H} \otimes \cdots \otimes \mathcal{H}$.

Note that, by Lemma 1.2, the complete $\beta$-boundedness condition can be equivalently formulated in terms of the von Neumann algebra $\mathcal{M}=\mathfrak{A}^{\prime \prime}$.

Corollary 1.10. Let $\mathcal{M}$ be a von Neumannalgebra acting cyclic and separating vector $\Omega \in \mathcal{H}$ and $U(t)=e^{i t K}$ a $\Omega$-fixing one-parameter unitary group implementing automorphisms of $\mathfrak{A}$. If $\mathcal{M}$ is completely $\beta$-bounded with respect to $K$ and $\Omega$, then

$$
2 \beta K=-T \log \Delta
$$

where $T$ is a positive linear operator, $0 \leq T \leq 1$, commuting with $\Delta, K$ and the modular conjugation $J$ of $(\mathcal{M}, \Omega)$.

Proof. By Corollary 1.8 applied to the $n$-fold tensor product, we have $e^{-2 \beta K} \otimes$ $\cdots \otimes e^{-2 \beta K} \leq 1+\Delta \otimes \cdots \otimes \Delta$. By the modular theory $K$ and $\Delta$ commute, thus a simple application of the Gelfand-Naimark theorem implies $e^{-2 n \beta K} \leq 1+\Delta^{n}$ for all $n$, thus $e^{-2 \beta K} \leq \sqrt[n]{1+\Delta^{n}}$. Taking the limit as $n \rightarrow \infty$ we obtain

$$
e^{-2 \beta K} \leq \max (1, \Delta) \text {. }
$$

As $J K J=-K$ and $J \log \Delta J=-\log \Delta$, the above inequality also entails that

$$
e^{2 \beta K} \leq \max \left(1, \Delta^{-1}\right) .
$$

Taking logarithms, as $K$ and $\Delta$ commute, these inequalities imply respectively $2 \beta K E_{+} \leq-\log \Delta E_{+}$and $2 \beta K E_{-} \geq-\log \Delta E_{-}$, where $E_{+/-}$are the spectral projections of $\log \Delta$ corresponding to the positive/negative half-line.

The projection $E_{0}$ onto the kernel of $\log \Delta$ clearly commutes with $\Delta$ and $K$, thus denoting by $T$ the closure of $-2 \beta K(\log \Delta)^{-1}\left(1-E_{0}\right), T$ is a bounded positive linear contraction, $0 \leq T \leq 1$, commuting both with $\Delta$ and $K$. As $J K J=-K$ and $J \log \Delta J=-\log \Delta$ and $J E_{0} J=E_{0}$, we also have $J T J=$ $T$.

We now recall a weak form of the characterization of the KMS property in terms of the Roepstoff-Araki-Sewell auto-correlation lower bound and the PuszWoronowicz passivity condition, see [7]. 
Theorem 1.11. 1, 33, 32]. Let $\mathcal{M}$ be a von Neumann algebra and $\Omega$ a cyclic and separating vector and $U(t)=e^{i t K}$ a $\Omega$-fixing one-parameter unitary group implementing automorphisms of $\mathcal{M}$. Consider following properties

(i) $(K X \Omega, X \Omega) \geq 0$ for all $X \in \mathcal{M}_{s a}$ in the domain of the derivation $[\cdot, K]$, where $\mathcal{M}_{\text {sa }}$ denotes the selfadjoint real subspace of $\mathcal{M}$.

(ii) $K=-\lambda \log \Delta$ for some $\lambda \in[0,+\infty)$.

Then $(i i) \Rightarrow(i)$. If the group of automorphisms of $\mathcal{M}$ preserving $(\cdot \Omega, \Omega)$ is ergodic then $(i) \Rightarrow(i i)$.

Note that case $(i)$ in the above theorem cannot hold with $K$ a non-trivial positive operator (ground state) because then $K$ is affiliated to $\mathcal{M}$ by a theorem of Borchers [6] (see also [26]), thus $K=0$ because $\Omega$ is separating.

We shall need to test the above inequality in $(i)$ for more vectors.

Lemma 1.12. Let $\mathcal{M}$ be a von Neumann algebra and $\Omega$ a cyclic and separating vector. Then

$$
-(\log \Delta \xi, \xi) \geq 0
$$

for all vectors $\xi \in \mathcal{K} \cap D(\log \Delta)$, where $\mathcal{K}$ is the real Hilbert subspace given by $\mathcal{K} \equiv \overline{\mathcal{M}_{s a} \Omega}$.

Proof. Let $S=J \Delta^{\frac{1}{2}}$ be the Tomita operator and $E_{n}$ be the spectral projection of $\log \Delta$ corresponding to the interval $(-n, n)$. Then $E_{n}$ commutes with the (real, unbounded) projection $P=\frac{1}{2}(1+S)$ onto $\mathcal{K}$ (see Lemma 1.13) because it commutes with $S=J \Delta^{\frac{1}{2}}$ : indeed $E_{n}$ commutes both with $\Delta$ and with $J$ (being a real even function of $\log \Delta$ ).

Denoting by $\mathcal{M}(-n, n)$ the space of elements of $\mathcal{M}$ whose spectrum under the modular group $\sigma_{t}=\operatorname{Ad} \Delta^{i t}$ lies in $(-n, n)$ and by $\mathcal{M}_{s a}(-n, n)$ the real subspace of selfadjoint elements of $\mathcal{M}(-n, n)$, we have

$$
\mathcal{M}_{s a}(-n, n) \Omega=\frac{1}{2}(1+S) \mathcal{M}(-n, n) \Omega=P \mathcal{M}(-n, n) \Omega .
$$

Let $\mathcal{H}_{S}$ denote the Hilbert space $D(S)$ equipped with the $S$-graph scalar product

$$
(\xi, \eta)_{S} \equiv(\xi, \eta)+(S \xi, S \eta)=(\xi, \eta)+\left(\Delta^{\frac{1}{2}} \xi, \Delta^{\frac{1}{2}} \eta\right), \xi, \eta \in D(S),
$$

and notice that any $\mathfrak{S}$ subset of $E_{n} \mathcal{H}$ is contained in $\mathcal{H}_{S}$ and its closure $\overline{\mathfrak{S}}$ in $\mathcal{H}$ coincides with its closure in $\mathcal{H}_{S}$ (because the restriction of $\Delta^{\frac{1}{2}}$ to $E_{n} \mathcal{H}$ is bounded).

Notice also that, as a linear operator of $\mathcal{H}_{S}, P$ is bounded, indeed $P$ is the (real) orthogonal projection of $\mathcal{H}_{S}$ onto $\mathcal{K}$.

Therefore, on the Hilbert space $\mathcal{H}_{S}$, we have by eq. (1.9) that

$$
\overline{\mathcal{M}_{s a}(-n, n) \Omega}=P \overline{\mathcal{M}(-n, n) \Omega}=P E_{n} \mathcal{H}_{S}=E_{n} P \mathcal{H}_{S}=E_{n} \mathcal{K} .
$$


As the the inequality (1.8) holds for all $\xi \in \mathcal{M}_{s a}(-n, n) \Omega$, it then holds for all $\xi \in E_{n} \mathcal{K}$.

Given $\xi \in \mathcal{K} \cap D(\log \Delta)$ the sequence of vectors $\xi_{n} \equiv E_{n} \xi$ belongs to $\overline{\mathcal{M}_{s a}(-n, n) \Omega}=E_{n} \mathcal{K}$ and

$$
\left\|\xi_{n}-\xi\right\| \rightarrow 0, \quad\left\|\log \Delta \xi_{n}-\log \Delta \xi\right\| \rightarrow 0,
$$

therefore

$$
-(\log \Delta \xi, \xi)=-\lim _{n}\left(\log \Delta \xi_{n}, \xi_{n}\right) \geq 0 .
$$

Lemma 1.13. Let $\mathcal{M}$ be a von Neumann algebra with a cyclic separating vector $\Omega$ and let $S$ be the associated Tomita's operator, i.e. the closure of $X \Omega \rightarrow X^{*} \Omega$, $X \in \mathcal{M}$. Then $\overline{\mathcal{M}_{s a} \Omega}=\{\xi \in D(S): S \xi=\xi\}$.

Proof. As is well known, $S^{*}=F$, where $F$ is the Tomita's operator associated with $\mathcal{M}^{\prime}$ and $\Omega$. With $\mathcal{K} \equiv \overline{\mathcal{M}_{s a} \Omega}$, let $\tilde{S}$ the operator given by $\tilde{S}: \xi+i \eta \rightarrow \xi-i \eta$, $\xi, \eta \in \overline{\mathcal{M}_{s a} \Omega}$, and define analogously $\tilde{F}$ with respect to $\overline{\mathcal{M}^{\prime}{ }_{s a} \Omega}$. Then $\tilde{S} \supset S$ and $\tilde{F} \supset F$ and $\tilde{S}^{*} \supset \tilde{F}$, thus $\tilde{S}=S$ and $\tilde{F}=F$.

For completeness we give a generalization of the inequality (1.8) that, at the same time, gives a direct proof of it.

Proposition 1.14. Let $\mathcal{H}$ be a complex Hilbert space and $\mathcal{K}$ a standard real Hilbert subspace, namely $\mathcal{K} \cap i \mathcal{K}=\{0\}, \overline{\mathcal{K}+i \mathcal{K}}=\mathcal{H}$. Then

$$
-(\log \Delta \xi, \xi) \geq 0
$$

for all vectors $\xi \in \mathcal{K} \cap D(\log \Delta)$, where $\Delta$ is the modular operator on $\mathcal{H}$ associated with $\mathcal{K}$.

Proof. Since the kernel of $\log \Delta$ is invariant under $J$ and $\Delta$, one can decompose $\mathcal{K}$ as a direct sum of two components, one corresponding to the kernel of $\log \Delta$, and one to its orthogonal complement, thus the inequality (1.10) can be proved for each component separately. Since the inequality is obviously satisfied on the kernel of $\log \Delta$, we may just suppose the kernel of $\log \Delta$ to be trivial.

Now we give an explicit description of the vectors of $\mathcal{K}$ (cf. [14]) which allows an immediate verification of the inequality 1.10.

Let us chse choose a selfadjoint antiunitary $C$ commuting with $J$ and $\Delta$, and set $U=J C$, so that $U \log \Delta U=-\log \Delta$. Then denote with $\mathcal{L}$ the real vector space of $C$-invariant vectors in the spectral subspace $\{\log \Delta>0\}$ and by $\psi^{ \pm}$ the maps $\psi^{+}: y \in \mathcal{L} \mapsto U \cos \Theta y+\sin \Theta y, \psi^{-}: y \in \mathcal{L} \mapsto i U \cos \Theta y-i \sin \Theta y$, where the operator $\Theta$ is defined by $|\log \Delta|=-2 \log \tan \Theta / 2, \sigma(\Theta) \subseteq[0, \pi / 2]$.

Since $U$ maps the spectral space $\{\log \Delta>0\}$ onto the spectral space $\{\log \Delta<$ $0\}$, both $\psi^{+}$and $\psi^{-}$are isometries, and a simple calculation shows that their ranges are real-orthogonal. Moreover, decomposing $\mathcal{H}$ as $\{\log \Delta<0\} \oplus\{\log \Delta>$ 
$0\}$, one can show that any solution of the equation $S x=x$ can be written as a sum $\psi^{+}(y)+\psi^{-}(z)$, namely the map $\psi^{-}+\psi^{+}: \mathcal{L} \oplus_{\mathbb{R}} \mathcal{L} \rightarrow \mathcal{K}$ is an isometric isomorphism of real Hilbert spaces.

Moreover, for any $y, z \in \mathcal{L},\left(\psi^{\mp}(y), \log \Delta \psi^{ \pm}(z)\right)$ is purely imaginary, therefore

$$
\begin{aligned}
\left(\left(\psi^{-}(y)+\psi^{+}(z)\right), \log \Delta\right. & \left.\left(\psi^{-}(y)+\psi^{+}(z)\right)\right) \\
& =\left(\psi^{-}(y), \log \Delta \psi^{-}(y)\right)+\left(\psi^{+}(z), \log \Delta \psi^{+}(z)\right),
\end{aligned}
$$

namely the inequality should be checked on $\psi^{+}(L)$ and $\psi^{-}(L)$ separately. Finally,

$$
\left(\psi^{-}(y), \log \Delta \psi^{-}(y)\right)=-(y, \cos \Theta \log \Delta y) \leq 0,
$$

since $\cos \Theta$ and $\log \Delta$ are commuting positive operators on $\mathcal{L}$, and the same holds on the range of $\psi^{+}$.

Theorem 1.15. Let $\mathcal{M}$ be a von Neumann algebra with a cyclic and separating vector $\Omega$ and $U(t)=e^{i t K}$ a $\Omega$-fixing one-parameter unitary group implementing automorphisms of $\mathcal{M}$. If $\mathcal{M}$ is completely $\beta$-bounded with respect to $K$ and $\Omega$, then either $K=0$ or $\operatorname{Ad} U(t)$ satisfies the $K M S$ condition at some inverse temperature $\beta_{0} \geq \beta$; indeed $\beta_{0}$ is the greatest $\beta>0$ such that $\mathcal{M}$ is completely $\beta$-bounded.

Proof. Set $2 \beta K=T \log \Delta$ as in Cor. 1.10. Note first that $\mathcal{K}=\overline{\mathcal{M}_{s a} \Omega}$ is equal to $\{\xi \in \mathcal{H}: S \xi=\xi\}$ where $S=J \Delta^{\frac{1}{2}}$ is the Tomita operator. As $T$ commutes both with $\Delta$ and $J$, the same is true for $T^{\frac{1}{2}}$ and thus $T^{\frac{1}{2}}$ commutes with $S$. It follows that

$$
T^{\frac{1}{2}} \mathcal{K} \subset \mathcal{K}
$$

We then have for all $\xi \in \mathcal{K}$

$$
2 \beta(K \xi, \xi)=(T \log \Delta \xi, \xi)=\left(\log \Delta T^{\frac{1}{2}} \xi, T^{\frac{1}{2}} \xi\right) \leq 0 .
$$

Clearly the same is true if we replace the von Neumann algebra $\mathcal{M}$ by $\otimes_{\mathbb{Z}} \mathcal{M}$ (infinite tensor product with respect to the constant sequence of vectors $\Omega_{n} \equiv$ $\Omega)$, the vector $\Omega$ by $\otimes_{\mathbb{Z}} \Omega_{n}$ and $U(t)$ by $\otimes_{\mathbb{Z}} U(t)$.

The permutation shift then acts in a strongly cluster fashion on the latter system. By the Pusz-Woronowicz theorem 1.11 either $K=0$ or this latter system satisfies the KMS condition at some inverse temperature $\beta>0$. Clearly the KMS condition then holds true also for the original system. To prove the last assertion we have to show that, given $\beta>1, \mathcal{M}$ is not completely $\beta$-bounded with respect to $-\log \Delta$ and $\Omega$. Indeed, if this were not the case, we would have $\Delta^{\beta} \leq \max (1, \Delta)$, by Theorem 1.3, which is not possible if $\beta>1$ unless $\Delta=1$ in which case also $K=0$. 
Let $\mathfrak{A}$ be a $\mathrm{C}^{*}$-algebra, $\alpha$ a one-parameter automorphism group and $\omega$ an $\alpha$-invariant state. At this point it is natural to say that $\omega$ is completely $\beta$ holomorphic if state $\omega \otimes \cdots \otimes \omega$ of the $n$-fold (spatial) tensor product $\mathfrak{A} \otimes \cdots \otimes \mathfrak{A}$ is $\beta$-holomorphic with constant $C=1$ for all $n \in \mathbb{N}$. We then have:

Theorem 1.16. Let $\mathfrak{A}$ be a $C^{*}$-algebra, $\alpha$ a non-trivial one-parameter automorphism group and $\omega$ an $\alpha$-invariant state. The following are equivalent:

(i) $\omega$ is completely $\beta$ holomorphic;

(ii) $\omega$ satisfies the KMS condition at inverse temperature $\beta_{\max }=\sup \{\beta>0$ : $\omega$ is completely $\beta$-holomorphic $\} \quad\left(\beta_{\max }=+\infty\right.$ means that $\omega$ is a ground state).

Proof. (ii) $\Rightarrow($ i $)$ : If $\omega$ satisfies the KMS condition at inverse temperature $\beta>0$ then it is $\beta$-holomorphic with constant $C=1$. It is also immediate by the inequality (1.2) that $\omega$ is not completely $\beta^{\prime}$-holomorphic if $\beta^{\prime}>\beta$. For the same reason it is not completely $\beta^{\prime}$-holomorphic if $\beta^{\prime}>\beta$.

If $\omega$ is a ground state, then $\omega$ is obviously completely $\beta$-holomorphic for all $\beta>0$.

$(i) \Rightarrow(i i)$ : By considering the GNS representation of $\omega$, it is sufficient to show that Theorem 1.15 holds true without assuming that $\Omega$ is separating, but allowing $\omega$ to be a ground state.

Indeed let $E \in \mathcal{M}$ be the projection onto $\mathcal{H}_{0}=\overline{\mathcal{M}^{\prime} \Omega}$. Clearly $E \mathcal{M} E$ is $\beta$-bounded on $\mathcal{H}_{0}$ with respect to $\left.K\right|_{\mathcal{H}_{0}}$ and $\Omega$. Hence $\left.\operatorname{Ad} U(t)\right|_{\mathcal{H}_{0}}$ implements the rescaled modular group of $(E \mathcal{M} E, \Omega)$. Thus $\beta^{\prime} K E=-\log \Delta$, for some $\beta^{\prime} \geq \beta$, where $\Delta$ is the modular operator of $E \mathcal{M} E$ acting on $\mathcal{H}_{0}$.

Moreover, by Corollary 1.8, we have $e^{-\beta K} \leq 1+\Delta E$. In particular $e^{-\beta K}(1-$ $E) \leq(1-E)$, thus $K(1-E) \geq 0$.

Thus $\beta^{\prime} K=-\log \Delta E+L$, where $L \equiv \beta^{\prime} K(1-E)$ is positive. By the completely $\beta$-holomorphic assumption, the same relation holds true by replacing the system with its tensor product by itself. This is possibly only in two cases: either $\log \Delta=0$, thus $K>0$ and $\omega$ is a ground state, or $L=0$, namely $\Omega$ is cyclic and separating and $\beta K=-\log \Delta$.

\subsubsection{Appendix. The domain of the analytic generator}

We collect here a few properties for selfadjoint operators needed in text.

Lemma 1.17. Let $K$ be a selfadjoint operator on the Hilbert space $\mathcal{H}, \beta>0$, and $\xi$ a vector in $\mathcal{H}$. The following are equivalent:

(i) $\xi \in D\left(e^{-\frac{\beta}{2} K}\right)$;

(ii) The function $t \in \mathbb{R} \rightarrow\left(e^{i t K} \xi, \xi\right)$ extends to a function continuous in $\bar{S}_{\beta}$ and holomorphic in $S_{\beta}$;

(iii) For all $\eta \in D\left(e^{-\frac{\beta}{2} K}\right)$, the function $t \in \mathbb{R} \rightarrow\left(e^{i t K} \xi, \eta\right)$ extends to a function in $A\left(S_{\beta}\right)$. 
In this case $\underset{t \rightarrow i \beta}{\operatorname{anal.cont}}\left(e^{i t K} \xi, \xi\right)=\left\|e^{-\frac{\beta}{2} K} \xi\right\|^{2}$.

Proof. Let $E(\lambda)$ be the family of projections associated with $K$ by the spectral theorem, namely $K=\int \lambda \mathrm{d} E(\lambda)$. Then $\xi \in D\left(e^{-\frac{\beta}{2} K}\right)$ if and only if $\int e^{-\lambda \beta} \mathrm{d}\|E(\lambda) \xi\|^{2}<\infty$, i.e. $e^{-\lambda \beta} \in L^{1}(\mu)$, where $\mu(V)=\int_{V} \mathrm{~d}(E(\lambda) \xi, \xi)$ is the finite Borel spectral measure associated with $\xi$. By the next Sublemma (with a change of sign of $\beta$ ) this holds iff $t \rightarrow \hat{\mu}(-t) \equiv \int e^{i t \lambda} \mathrm{d} \mu(\lambda)$ is the boundary value of a function holomorphic in $S_{\beta}$ and continuous in $\bar{S}_{\beta}$ therefore $(i) \Leftrightarrow(i i)$.

If (ii) holds, then $t \rightarrow \hat{\mu}(-t)$ extends to a function in $A\left(S_{\beta}\right)$, namely $\hat{\mu}(-t)$ is bounded in the strip $S_{\beta}$, because

$$
\left|\int e^{i z \lambda} \mathrm{d} \mu(\lambda)\right| \leq \int e^{-\Im z \lambda} \mathrm{d} \mu(\lambda) \leq \mu([0, \infty))+\int e^{-\beta \lambda} \mathrm{d} \mu(\lambda)
$$

for all $z \in \bar{S}_{\beta}$.

If $\xi, \eta \in D\left(e^{-\frac{\beta}{2} K}\right)$, the function

$$
f(z) \equiv\left(e^{i t K} e^{-s K} \xi, e^{-s K} \eta\right), \quad z=t+i s \in \bar{S}_{\beta},
$$

can be checked to belong to $A\left(S_{\beta}\right)$ by standard methods, thus $(i i) \Leftrightarrow(i i i)$. Last assertion follows by the spectral theorem.

Sublemma 1.18. Let $\mu$ be a finite Borel measure on $\mathbb{R}, \hat{\mu}$ its Fourier transform and $\beta \in \mathbb{R}$. The function $t \in \mathbb{R} \rightarrow e^{\beta t}$ belongs to $L^{1}(\mu)$ if and only if $\hat{\mu}$ is the boundary value of a function holomorphic in $S_{\beta}$ and continuous in $\bar{S}_{\beta}$.

This function is automatically bounded, namely it belongs to $A\left(S_{\beta}\right)$.

Proof. If $\lambda \in \mathbb{R} \rightarrow e^{\beta \lambda}$ belongs to $L^{1}(\mu)$, then also $\lambda \in \mathbb{R} \rightarrow e^{-i z \lambda}$ belongs to $L^{1}(\mu)$ for all $0 \leq \Im z \leq \beta$ and $\hat{\mu}(z)=\int e^{-i z \lambda} \mathrm{d} \mu(\lambda)$ defines a function in the strip $\bar{S}_{\beta}$, that can be easily seen to belong to $A\left(S_{\beta}\right)$.

Conversely suppose $\hat{\mu}$ to be the boundary value of a function holomorphic in $S_{\beta}$ and continuous in $\bar{S}_{\beta}$. Decompose $\mu$ as $\mu_{+}+\mu_{-}$, where the first term is supported in the positive axis, the second in the negative axis. We have $e^{\beta t} \in L^{1}\left(\mathbb{R}, \mu_{-}\right)$for any positive $\beta$, hence $\hat{\mu}_{-}$is holomorphic in the upper half plane. Therefore we may restrict to the case where $\mu$ is supported in the positive axis.

In this case $\hat{\mu}$ is holomorphic in the lower half plane and in the strip $S_{\beta}$, and is continuous on the real line both from above and from below, therefore $\hat{\mu}$ extends to a holomorphic function on $\{\Im z<\beta\}$, continuous on the boundary. Set $\varphi(x)=\hat{\mu}(i x)$. Then $\varphi$ is analytic in $x<\beta$, continuous on $x \leq \beta$, and for $x \leq 0$ is given by $\varphi(x)=\int e^{x \lambda} \mathrm{d} \mu(\lambda)$. If $x<0$, the dominated convergence theorems entails $\varphi^{(n)}(x)=\int \lambda^{n} e^{x \lambda} \mathrm{d} \mu(\lambda)$, hence by monotone convergence we obtain $\varphi^{(n)}(0)=\int \lambda^{n} \mathrm{~d} \mu(\lambda)$. The analyticity implies that for $0 \leq x<\beta$

$$
\varphi(x)=\sum_{n=0}^{\infty} \frac{x^{n}}{n !} \int \lambda^{n} \mathrm{~d} \mu(\lambda)=\int e^{x \lambda} \mathrm{d} \mu(\lambda)
$$


where the last equality follows by monotone convergence. Again by monotone convergence and the continuity of $\varphi$ on the boundary we get $\varphi(\beta)=\int e^{\beta \lambda} \mathrm{d} \mu(\lambda)$, namely $e^{\beta t} \in L^{1}(\mu)$.

We shall also need the following proposition.

Proposition 1.19. Let $U(t)=e^{i t K}$ be a one-parameter unitary group on a Hilbert space $\mathcal{H}$ and $\varphi: \mathbb{R} \rightarrow \mathbb{C}$ a locally bounded Borel function. If $\mathcal{D} \subset$ $D(\varphi(K))$ is a dense, $U$-invariant linear space, then $\mathcal{D}$ is a core for $\varphi(K)$.

Proof. By replacing $\varphi$ with $|\varphi|$, we may assume that $\varphi$ is non-negative. Let $\xi \in \mathcal{H}$ be a vector orthogonal to $(\varphi(K)+1) \mathcal{D}$. We have to show that $\xi=0$. If $f$ is a function in the Schwartz space $S(\mathbb{R})$, we have

$$
((\varphi(K)+1) f(K) \eta, \xi)=\int \tilde{f}(t)\left((\varphi(K)+1) e^{-i t K} \eta, \xi\right) \mathrm{d} t=0,
$$

for all $\eta \in \mathcal{D}$, where $\tilde{f}$ the Fourier anti-transform of $f$.

If $f$ is a bounded Borel function with compact support, we may choose a sequence of smooth functions $f_{n}$ with compact support such that $f_{n}(K) \rightarrow f(K)$ weakly, thus eq. (1.12) holds for such an $f$.

If $g$ is a bounded Borel function with compact support, we may write $g(\lambda)=$ $(\varphi(\lambda)+1)(\varphi(\lambda)+1)^{-1} g(\lambda)$, therefore

$$
(g(K) \eta, \xi)=0 \text {. }
$$

We can then choose a sequence $g_{n}$ of such functions such that $g_{n}(K) \rightarrow 1$ strongly. It follows that $(\eta, \xi)=0$ for all $\eta \in \mathcal{D}$, hence $\xi=0$ because $\mathcal{D}$ is dense.

\subsection{Case where further symmetries are present}

We now examine the case where the one-parameter unitary group $\alpha$ considered before extends to a unitary representation of the " $a x+b$ " group, where positive translations implement endomorphisms of the algebra.

Proposition 1.20. Let $\mathcal{M}$ be a von Neumann algebra on the Hilbert space $\mathcal{H}$, $\Omega$ a cyclic vector for $\mathcal{M}$ and $U$ a $\Omega$-fixing one-parameter unitary group on $\mathcal{H}$, with generator $K$, implementing automorphisms of $\mathcal{M}$. Assume furthermore that there is a one-parameter unitary group $T$ on $\mathcal{H}$ such that

$$
T(a) \mathcal{M T}(-a) \subset \mathcal{M}, \quad \forall a \geq 0
$$

and satisfying the commutation relations

$$
U(t) T(a) U(-t)=T\left(e^{t} a\right), \quad a, t \in \mathbb{R} .
$$

If there is a dense ${ }^{*}$-subalgebra $\mathfrak{B}$ of $\mathcal{M}$ with such that $\mathfrak{B} \Omega \subset D\left(e^{-\pi K}\right)$ and either

- $U(t) \mathfrak{B} U(-t)=\mathfrak{B}$ and $T(a) \mathfrak{B} T(-a) \subset \mathfrak{B}, t \in \mathbb{R}, a \in \mathbb{R}^{+}$, or

- $\mathfrak{B}$ is $\pi$-bounded with respect to $K$ and $\Omega$, then the generator $H$ of $T$ is positive. 
Proof. Note first that, as a consequence of the commutation relations (1.13), we have $T(a) \Omega=\Omega$ for all $a \in \mathbb{R}$ [17]. By the the criterion given in Proposition 1.25 below, it will suffice to construct a core $\mathcal{D}$ for $e^{-\pi K}$ such that $T(a) \mathcal{D} \subset \mathcal{D}$ for all $a \geq 0$.

The set $\mathcal{D} \equiv \mathfrak{B} \Omega$ is contained in the domain of $e^{-\pi K}$, and clearly

$$
T(a) \mathcal{D}=T(a) \mathfrak{B} T(-a) \Omega \subset \mathfrak{B} \Omega=\mathcal{D}, \quad a \geq 0 .
$$

If moreover $\mathfrak{B}$ is $\operatorname{Ad} U$-invariant, then

$$
U(t) \mathcal{D}=U(t) \mathfrak{B} U(-t) \Omega=\mathfrak{B} \Omega=\mathcal{D}, \quad t \in \mathbb{R} .
$$

We may thus apply Lemma 1.24 below to conclude that $\mathcal{D}$ is a core for $e^{-\pi K}$.

On the other hand, if $\mathfrak{B}$ is $\pi$-bounded, then also $\mathcal{M}$ is $\pi$-bounded by Lemma 1.2. thus we are in the previous case as $\mathcal{M}$ is $\operatorname{Ad} U$-invariant.

Corollary 1.21. In the previous Prop. 1.20, suppose that $\mathfrak{B}$ is $\pi$-bounded and further that $\Omega$ is separating for $\mathcal{M}$ and $\mathbb{C} \Omega$ are the only $T$-invariant vectors.

Then $\omega \equiv(\cdot \Omega, \Omega)$ is a KMS state for $\alpha=\operatorname{Ad} U$ at inverse temperature $\beta=2 \pi$. Namely the modular operator associated with $(\mathcal{M}, \Omega)$ is $\Delta=e^{-2 \pi K}$.

Proof. Since $U$ implements automorphisms of $\mathcal{M}$, it commutes with the modular operator $\Delta$ associated with $(\mathcal{M}, \Omega)$, thus $U(2 \pi t) \Delta^{i t}$ is a one-parameter group of unitaries. We denote by $L$ its self-adjoint generator.

By the modular theory $U$ also commutes with the modular conjugation $J$ associated with $(\mathcal{M}, \Omega)$, thus $J e^{\pi K} J=e^{-\pi K}$. We then have, for all $X \in \mathcal{M}_{1}$,

$$
\left\|e^{-\pi L} X \Omega\right\|=\left\|e^{\pi K} \Delta^{1 / 2} X \Omega\right\|=\left\|e^{\pi K} J X^{*} \Omega\right\|=\left\|J e^{-\pi K} X^{*} \Omega\right\| \leq C .
$$

We shall now show that the above bound holds for all $X$ in the unit ball of the *-algebra $\mathfrak{C} \equiv \cup_{a} T(a) \mathcal{M} T(-a)$.

By Borchers theorem [5], $\Delta^{i t}$ has the same commutation relations (1.13) as $U(t)$ with $T(a)$, thus $e^{i t L}$ commutes with $T(a)$. Therefore if $X \in \mathcal{M}_{1}$

$$
\begin{aligned}
& \left\|e^{-\pi L} T(a) X T(-a) \Omega\right\|=\left\|e^{-\pi L} T(a) X \Omega\right\| \\
& \quad=\left\|T(a) e^{-\pi L} X \Omega\right\|=\left\|e^{-\pi L} X \Omega\right\| \leq C,
\end{aligned}
$$

namely the $\pi$-boundedness property with respect to $L$ holds for $\mathfrak{C}$.

Now, by the following Lemma $1.23, \mathfrak{C}$ is irreducible on $\mathcal{H}$, thus $L$ is semibounded by Cor. 1.3. As $J L J=-L, L$ is indeed a bounded operator.

We now follow an argument in [10]. By the Kadison-Sakai derivation theorem (cf. [38]), there exists a selfadjoint element $h \in \mathcal{M}$, indeed a minimal positive one, such that

$$
e^{i t h} X e^{-i t h}=e^{i t L} X e^{-i t L}, \quad X \in \mathcal{M},
$$

and indeed $\Delta^{i t} h \Delta^{-i t}=h$ by the canonicity of the minimal positive choice for $h$. Therefore $\Delta^{i t} h \Omega=h \Omega, t \in \mathbb{R}$, and this implies $T(a) h \Omega=h \Omega, a \in \mathbb{R}$ [17], thus $h \Omega \in \mathbb{C} \Omega$ by the uniqueness of the $T$-invariant vector. As $\Omega$ is separating, $h \in \mathbb{R}^{+}$, thus $h=0$ as $h$ is minimal.

It follows that $\Delta^{i t}=U(-2 \pi t)$ for all $t \in \mathbb{R}$. 
Note that, by an argument of Driessler, see [26], the von Neumann algebra $\mathcal{M}$ in the above corollary is a $I I I_{1}$-factor, unless $\operatorname{dim} \mathcal{H} \leq 1$.

\subsubsection{Appendix. Spectral and irreducibility properties}

We begin to recall a simple lemma.

Lemma 1.22. [12]. Let $\mathfrak{C}$ be $a^{*}$-algebra on a Hilbert space $\mathcal{H}$ with cyclic vector $\Omega$ and $E$ be the one-dimensional projection onto $\mathbb{C} \Omega$. The ${ }^{*}$-algebra generated by $\mathfrak{C}$ and $E$ is irreducible.

Proof. Let $X \in B(\mathcal{H})$ commute with $\mathfrak{C}$ and $E$. Then $X \in \mathfrak{C}^{\prime}$ and $X \Omega=X E \Omega=$ $E X \Omega=\lambda \Omega$ for some $\lambda \in \mathbb{C}$. As $\Omega$ is separating for $\mathfrak{C}^{\prime \prime}$, then $X=\lambda$ and this entails the thesis.

Lemma 1.23. [26] Let $\mathfrak{C}$ be $a^{*}$-algebra on a Hilbert space $\mathcal{H}, T$ a n-parameter unitary group, $n \geq 1$, such that $T(x) \mathfrak{C} T(-x)=\mathfrak{C}$ for $x \in \mathbb{R}^{n}$. If the spectrum of $T$ is asymmetric, namely $\operatorname{sp}(U) \cap-\operatorname{sp}(U)=\{0\}$ and $\Omega$ is a vector which is cyclic for $\mathfrak{C}$ and unique $T$-invariant, then $\mathfrak{C}$ is irreducible.

Proof. Let $\mathcal{M}$ be the weak closure of $\mathfrak{C}$. Clearly $T(x) \mathcal{M} T(-x)=\mathcal{M}$, hence, by a theorem of Borchers, [6], see also [15], $T(x) \in \mathcal{M}$.

By the mean ergodic theorem, the one dimensional projection $E$ onto $\Omega$ belongs to von Neumann algebra generated by $\left\{T(x), x \in \mathbb{R}^{n}\right\}$, hence to $\mathcal{M}$.

Then $\mathcal{M}=B(\mathcal{H})$ by the Lemma 1.22 .

Lemma 1.24. Let $\mathfrak{B}$ be $a^{*}$-algebra, $\Omega$ a cyclic vector for $\mathfrak{B}$ and $U(t)=e^{i t K}$ a one-parameter, $\Omega$-fixing unitary group implementing automorphisms of $\mathfrak{B}$. If $\mathfrak{B} \Omega \subset D\left(e^{-\beta K}\right)$ for some $\beta>0$, then $\mathfrak{B} \Omega$ is a core for $e^{-\beta K}$.

Proof. Set $\mathcal{D}=\mathfrak{B} \Omega$ and apply Proposition 1.19.

We now recall the criterion for the positivity of the energy discussed in [3].

Proposition 1.25. [3]. Let $U$ and $T$ be one-parameter unitary group on a Hilbert space $\mathcal{H}$ satisfying the commutation relations (1.13). The following are equivalent:

(i) the generator of $T$ is positive;

(ii) there exists a core $\mathcal{D}$ for $e^{-\pi K}$ such that $T(a) \mathcal{D} \subset \mathcal{D}$ for some (hence for all) $a>0$, where $K$ is the generator of $U$.

\section{Minimality of the Hawking temperature}

We now apply our results in the Quantum Field Theory context. 


\subsection{A characterization of the Bisognano-Wichmann prop- erty}

In the following we shall consider a Poincaré covariant net of von Neumann algebras on the Minkowski spacetime in the vacuum representation, indeed an inclusion preserving map

$$
\mathcal{S} \rightarrow \mathcal{A}(\mathcal{S})
$$

associating a von Neumann algebra acting on a given Hilbert space $\mathcal{H}$ with each spacelike cone $\mathcal{S}$ in the Minkowski spacetime $\mathbb{R}^{d+1}, d \geq 1$, satisfying the following properties:

- There exists a representation $U$ of the Poincaré group $\mathcal{P}_{+}^{\uparrow}$ such that

$$
U(g) \mathcal{A}(\mathcal{S}) U(g)^{*}=\mathcal{A}(g \mathcal{S}), \quad g \in \mathcal{P}_{+}^{\uparrow} .
$$

- There exists a unique unit vector $\Omega$ which is invariant under the action of the Poincaré group. $\Omega$ is cyclic and separating for the von Neumann algebras associated with wedge regions.

Here a spacelike cone $\mathcal{S}$ is the cone generated by double cone and a point in the interior of its spacelike complement.

Note that we do neither assume $\mathcal{A}$ to be local nor the positivity of the energy to hold. This last property will indeed follow by our boundedness condition.

With each wedge region we associate the one-parameter group $\Lambda_{W}$ of Lorentz boosts preserving $W$. In this way, denoting by $\Delta_{W}$ the Tomita operator associated with the von Neumann algebra $\mathcal{A}(W)$ and $\Omega$, the Bisognano-Wichmann relations 纤 take the form

$$
\Delta_{W}^{i t}=U\left(\Lambda_{W}(-2 \pi t)\right),
$$

where $W$ is a wedge. Clearly, by Poincaré covariance, eq. (2.1) holds for all wedges if it holds for a particular one. If $W$ is a wedge we denote by $K_{W}$ the generator of the one-parameter unitary group $U(\Lambda(t))$.

We now need the following geometric observation, whose proof is straightforward:

Lemma 2.1. If $\mathcal{S}$ is an open convex cone of $\mathbb{R}^{n}$, the set of its translated $\{\mathcal{S}+x$ : $\left.x \in \mathbb{R}^{n}\right\}$ is directed with respect to inclusion.

Theorem 2.2. Let $W$ be a wedge, $\mathcal{S}$ a spacelike cone contained in $W$ and $\mathfrak{B}$ a weakly dense *-subalgebra of $\mathcal{A}(\mathcal{S})$. The following are equivalent:

(i) The Bisognano-Wichmann relation $\Delta_{W}=e^{-2 \pi K_{W}}$ holds.

(ii) $e^{-\pi K_{W}} \mathfrak{B}_{1} \Omega$ is bounded and the energy-momentum spectrum lays in the forward light cone $\bar{V}_{+}$.

(iii) $e^{-\pi K_{W}} \mathcal{A}(W)_{1} \Omega$ is bounded 
(iv) $\left\|e^{-\pi K_{W}} \mathcal{A}(W)_{1} \Omega\right\| \leq 1$.

If moreover the boundary of $\mathcal{S}$ intersects the edge of $W$ in a half-line, then in (ii) the $\pi$-boundedness is sufficient, namely it implies the spectrum condition.

Proof. In this proof we drop the subscript $W$ on the operators associated with $W$.

$(i) \Rightarrow(i v):$ As $\Delta^{1 / 2}=e^{-\pi K}$, then $e^{-\pi K} X \Omega=\Delta^{1 / 2} X \Omega=J X^{*} \Omega$ for all $X \in \mathcal{A}(W)$, which immediately implies $(i v)$.

$(i v) \Rightarrow(i i i)$ is obvious.

(iii) $\Rightarrow($ ii $)$ : We only need to show the spectrum condition. By Poincarè covariance it is sufficient to show that the positivity of the generator of a oneparameter group $T$ of light-like translations associated with $W$; this satisfies $T(a) \mathcal{A}(W) T(-a) \subset \mathcal{A}(W), a \geq 0$, and the commutation relations (1.13) with $U(t) \equiv e^{i t K}$.

Thus the positivity property follows by the criterion in Proposition 1.25.

(ii) $\Rightarrow(i)$ : Since $U(\Lambda(t))$ implements automorphisms of $\mathcal{A}(W)$, it commutes with $\Delta^{i s}$, i.e. $U(\Lambda(2 \pi t)) \Delta^{i t}$ is a one-parameter group of unitaries. Denote by $L$ its self-adjoint generator and note that, since by the Tomita-Takesaki theorem $e^{i t L}$ commutes with the modular conjugation $J$ of $(\mathcal{A}(W), \Omega)$, we have for all $X \in \mathcal{A}(\mathcal{S})_{1}$

$$
\left\|e^{-L / 2} X \Omega\right\|=\left\|e^{-\pi K} \Delta^{1 / 2} X \Omega\right\|=\left\|e^{-\pi K} X^{*} \Omega\right\| \leq C,
$$

namely $\mathcal{A}(\mathcal{S})$ is $\frac{1}{2}$-bounded with respect to $L$ and $\Omega$.

Moreover, by Borchers commutation relations [5] and by Tomita-Takesaki theorem, $e^{i t L}$ commutes with all translations. Therefore, denoting by $T$ the translation unitary group,

$$
\left\|e^{-L / 2} \mathcal{A}(\mathcal{S}+x)_{1} \Omega\right\|=\left\|e^{-L / 2} T(x) \mathcal{A}(\mathcal{S})_{1} \Omega\right\|=\left\|e^{-L / 2} \mathcal{A}(\mathcal{S})_{1} \Omega\right\| \leq C,
$$

namely $\left\|e^{-L / 2} X \Omega\right\| \leq C$ for all $X \in \cup_{x} \mathcal{A}(\mathcal{S}+x)$ with $\|X\| \leq 1$.

By Lemma 2.1 $\cup_{x} \mathcal{A}(\mathcal{S}+x)$ is a ${ }^{*}$-subalgebra of $\mathcal{B}(\mathcal{H})$, which is also translation invariant. By Lemma $1.23 \cup_{x} \mathcal{A}(\mathcal{S}+x)$ is thus irreducible, thus $L$ is semibounded by lemma 1.3 .

The rest now follows as in the proof of Corollary 1.21.

It remains to show the last assertion. Let's then assume that $\mathcal{S}$ intersects the edge of $W$ in a half line.

By Lemma 1.2 the boundedness of $e^{-\pi K} \mathfrak{B}_{1} \Omega$ implies that also $e^{-\pi K} \mathcal{A}(\mathcal{S})_{1} \Omega$ is bounded.

Let $T(s)$ be the one-parameter unitary group of translations along the edge of $W$. Clearly $T(s)$ commutes with $K$, therefore if $\left\|e^{-\pi K} \mathcal{A}(\mathcal{S})_{1} \Omega\right\| \leq C$ then

$$
\begin{aligned}
\left\|e^{-\pi K} \mathcal{A}(\mathcal{S}+s)_{1} \Omega\right\| & =\left\|e^{-\pi K} T(s) \mathcal{A}(\mathcal{S})_{1} \Omega\right\| \\
& =\left\|T(s) e^{-\pi K} \mathcal{A}(\mathcal{S})_{1} \Omega\right\|=\left\|e^{-\pi K} \mathcal{A}(\mathcal{S})_{1} \Omega\right\| \leq C,
\end{aligned}
$$

namely the $\pi$-boundedness condition hold for $\cup_{s} \mathcal{A}(\mathcal{S}+s)$. As $\mathcal{A}(\mathcal{S}+s)$ is a dense *-algebra of $\mathcal{A}(W)$, by Lemma $1.2\left\|e^{-\pi K} \mathcal{A}(W)_{1} \Omega\right\| \leq C$, thus we obtain all the properties in the statement by the above proof. 
Let now $\mathcal{M}$ be a von Neumann algebra on a Hilbert space $\mathcal{H}$ and $\Omega$ a cyclic and separating vector for $\mathcal{M}$. For a vector $\xi \in \mathcal{H}$ we set $\left.\varphi_{\xi} \equiv(\cdot \Omega, \xi)\right|_{\mathcal{M}}$. We shall say that a set $Q \subset \mathcal{H}$ is $L^{1}$-metrically nuclear with respect to $\mathcal{M}$ if the set of linear functionals $\left\{\varphi_{\xi} \in \mathcal{M}_{*}: \xi \in Q\right\}$ is a metrically nuclear subset of $\mathcal{M}_{*}$.

By using a characterization of the split property of Fidaleo [13], we obtain the following.

Corollary 2.3. Let $W$ be a wedge region and $\mathcal{S}$ a spacelike cone contained in $W$. Consider the following properties:

(i) The Bisognano-Wichmann property $\Delta=e^{-2 \pi K_{W}}$ for $W$;

(ii) The split property for $\mathcal{A}(\mathcal{S}) \subset \mathcal{A}(W)$.

(iii) The set $e^{-\lambda K_{W}} \mathcal{A}(\mathcal{S})_{1} \Omega$ is compact for every $0<\lambda<\pi, L^{1}$-metrically nuclear with respect to $\mathcal{A}(W)$ for $\lambda=1 / 2$, and the diameter of $e^{-\lambda K_{W}} \mathcal{A}(\mathcal{S})_{1} \Omega$ is uniformly bounded for $0<\lambda<\pi$.

Then $(i) \&(i i) \Leftrightarrow(i i i)$.

Proof. $(i) \&(i i) \Rightarrow($ iii $)$ : By the split property the set $\Delta^{\lambda} \mathcal{A}(\mathcal{S})_{1} \Omega$ is compact for $0<\lambda<1 / 2$ [8] and metrically nuclear for $\lambda=1 / 4$ [13]. By the KMS property $\left\|\Delta^{\lambda} X \Omega\right\| \leq 1$ for all $X \in \mathcal{A}(W)_{1}$ and $0 \leq \lambda \leq \frac{1}{2}$ (see [ [ ] ); we omit the suffix $W$ on $\Delta$ and $K$.

$($ iii $) \Rightarrow(i) \&(i i)$ : Assume first that the underlying Hilbert $\mathcal{H}$ space is separable. Let $X \in \mathcal{A}(\mathcal{S})_{1}$ and choose a sequence $\left\{\xi_{n}\right\}$ norm dense in $\mathcal{H}_{1}$; the function

$$
F_{n}(z) \equiv\left(e^{i z K} X \Omega, \xi_{n}\right)
$$

is bounded and holomorphic in the open strip $S_{\pi}$. Indeed, by the uniform boundedness assumption, $|F(z)| \leq C$ for some constant $C>0$ independent of $X$ and $\xi_{n}$.

As $F_{n} \in H^{\infty}\left(S_{\pi}\right)$ the limit $\lim _{\lambda \rightarrow \pi} F_{n}(t+i \lambda)$ exists except for $t$ in a set $E_{n} \subset$ $\mathbb{R}$ of Lebesgue measure zero. Choose $t_{0} \notin \cup_{n} E_{n}$; as $\left\|e^{i\left(t_{0}+i \lambda\right) K} X \Omega\right\| \leq C$, the

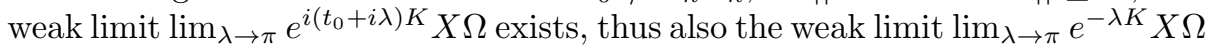
exists. By the spectral theorem, this implies $X \Omega \in D\left(e^{-\pi K}\right)$ and $\left\|e^{-\pi K} X \Omega\right\| \leq$ $C$, namely $\mathcal{A}(\mathcal{S})$ is $\pi$-bounded with respect to $K$ and $\Omega$ and this entails $(i)$ by the previous theorem. If $\mathcal{H}$ is non-separable, it is sufficient to apply the above argument to the separable Hilbert subspace generated by $f(K) X \Omega$ as $f$ varies in the complex continuous functions on $\mathbb{R}$ vanishing at infinity.

Then (ii) follows because the $L^{1}$-metrical nuclearity of $\Delta^{1 / 2} \mathcal{A}(\mathcal{S})_{1} \Omega$ implies the split property for $\mathcal{A}(\mathcal{S}) \subset \mathcal{A}(W)[13$.

Remark 2.4. If the net $\mathcal{A}$ is local, then the modular conjugation $J_{W}$ for wedges has a geometric action too [16] if Theorem 2.2 holds. 


\subsection{One-dimensional nets}

It is convenient to give explicitly a version of the above results in the context of nets of von Neumann algebras on the real line.

Let $\mathcal{I}$ denote the set of bounded open non-empty intervals of $\mathbb{R}$. We shall consider a net of von Neumann algebras on $\mathbb{R}$, namely an inclusion preserving map

$$
I \in \mathcal{I} \rightarrow \mathcal{C}(I)
$$

from $\mathcal{I}$ to the von Neumann algebras on a given Hilbert space $\mathcal{H}$. If $E \subset \mathbb{R}$, we denote by $\mathfrak{C}(E)$ the $\mathrm{C}^{*}$-algebra generated by $\{\mathcal{C}(I): I \in \mathcal{I}, I \subset E\}$ and by $\mathcal{C}(E)$ the weak closure of $\mathfrak{C}(E)$.

We shall further assume that there exist two one-parameter unitary groups $T$ and $U$ implementing translations and dilations, namely for any $I \in \mathcal{I}, a, t \in \mathbb{R}$,

$$
\begin{aligned}
T(a) \mathcal{C}(I) T(a)^{*} & =\mathcal{C}(I+a) \\
U(t) \mathcal{C}(I) U(t)^{*} & =\mathcal{C}\left(e^{t} I\right)
\end{aligned}
$$

satisfying the commutation relations $(1.13)$ and leaving invariant a unique unit vector $\Omega$ (the vacuum), which is cyclic and separating for $\mathcal{C}(0, \infty)$.

We denote by $K$ the infinitesimal generator of $U$.

Proposition 2.5. The following are equivalent:

(i) The Bisognano-Wichmann relations $\Delta=e^{-2 \pi K}$ holds, where $\Delta$ is the modular operator associated with $(\mathcal{C}(0, \infty), \Omega)$.

(ii) There exists $a>0$ and a dense ${ }^{*}$-subalgebra $\mathfrak{B}$ of $\mathcal{C}(0, a)$ such that $e^{-\pi K} \mathfrak{B}_{1} \Omega$ is bounded.

(iii) There exists $a>0$ and a dense ${ }^{*}$-subalgebra $\mathfrak{B}$ of $\mathcal{C}(a, \infty)$ such that $e^{-\pi K} \mathfrak{B}_{1} \Omega$ is bounded.

(iv) There exists $I \in \mathcal{I}$ and a dense ${ }^{*}$-subalgebra $\mathfrak{B}$ of $\mathcal{C}(I)$ such that $e^{-\pi K} \mathfrak{B}_{1} \Omega$ is bounded and the generator of $T$ is a positive operator.

(v) $e^{-\pi K} \mathcal{C}(0, \infty)_{1} \Omega$ is a bounded set.

Proof. The proof is similar to the one of Theorem 2.2. We only notice that in this case condition (ii) refers to a bounded interval $(0, a)$. This is possible because $\left\|e^{-\pi K} \mathfrak{B}_{1} \Omega\right\| \leq C \Rightarrow\left\|e^{-\pi K} \mathcal{C}(0, a)_{1} \Omega\right\| \leq C$ and, by scaling the interval, this gives

$$
\left\|e^{-\pi K} \mathcal{C}\left(0, e^{t} a\right)_{1} \Omega\right\|=\left\|e^{i t K} e^{-\pi K} \mathcal{C}(0, a)_{1} \Omega\right\|=\left\|e^{-\pi K} \mathcal{C}(0, a)_{1} \Omega\right\| \leq C
$$

for any $t$, thus the boundedness condition holds for $\mathfrak{C}(0, \infty)$.

Remark 2.6. If locality is further assumed in Prop. 2.5, then the net $\mathcal{C}$ extends to a conformal net on $S^{1}$ [18]. 


\subsection{Globally hyperbolic spacetimes}

We shall now discuss our results for a class of stationary black hole spacetimes, namely globally hyperbolic spacetimes with bifurcate Killing horizon. As we shall see, for nets with a boundedness property the KMS property is equivalent to the existence of a translation symmetry for the net on the horizon.

Let $\mathcal{V}$ be a $d+1$ dimensional globally hyperbolic spacetime with a bifurcate Killing horizon. An example is given by the Schwarzschild-Kruskal manifold. We denote by $\mathfrak{h}_{+}$and $\mathfrak{h}_{-}$the two codimension 1 submanifolds that constitute the horizon $\mathfrak{h}=\mathfrak{h}_{+} \cup \mathfrak{h}_{-}$. We denote by $\mathcal{L}$ and $\mathcal{R}$ the "left and right wedges".

Let $\kappa=\kappa(\mathcal{V})$ be the surface gravity, namely, denoting by $\chi$ the Killing vector field, the equation $\nabla g(\chi, \chi)=-2 \kappa \chi$ on $\mathfrak{h}$, with $g$ the metric tensor, defines a function $\kappa$ on $\mathfrak{h}$, that is actually constant on $\mathfrak{h}$ 25. If $\mathcal{V}$ is the SchwarzschildKruskal manifold, then $\kappa(\mathcal{V})=\frac{1}{4 m}$, where $m$ is the mass of the black hole. In this case $\mathcal{R}$ is the exterior of the Schwarzschild black hole.

In what follows $\mathcal{R}$ is the actual spacetime, and $\mathcal{V}$ is to be regarded as a completion of $\mathcal{R}$.

Let $\mathcal{A}(\mathcal{O})$ be the von Neumann algebra on a Hilbert space $\mathcal{H}$ of the observables localized in the bounded diamond $\mathcal{O} \subset \mathcal{R} . \mathcal{R} \subset \mathcal{V}$ is a $\Lambda$-invariant region and we assume that the Killing flow $\Lambda_{t}$ of $\mathcal{V}$ gives rise to a one parameter unitary group $U(t)=e^{i K t}$ implementing automorphisms $\alpha_{t}$ of the quasi-local $C^{*}$-algebra $\mathfrak{A}(\mathcal{R})$ such that $\alpha_{t}(\mathcal{A}(\mathcal{O}))=\mathcal{A}\left(\Lambda_{t}(\mathcal{O})\right)$.

We now consider a locally normal $\alpha$-invariant state $\varphi$ on $\mathfrak{A}(\mathcal{R})$. The net $\mathfrak{A}$ is assumed to be already in the GNS representation of $\varphi$, hence $\varphi$ is represented by a cyclic vector $\xi$. Let's denote by $\mathcal{R}_{a}$ the wedge $\mathcal{R}$ "shifted by" $a \in \mathbb{R}$ along, say, $\mathfrak{h}_{+}$(see [19]). If $I=(a, b)$ is a bounded interval contained in $\mathbb{R}_{+}$, we set

$$
\mathcal{C}(I)=\mathfrak{A}\left(\mathcal{R}_{a}\right)^{\prime \prime} \cap \mathfrak{A}\left(\mathcal{R}_{b}\right)^{\prime}, \quad 0<a<b .
$$

One obtains in this way a net of von Neumann algebras localized on the horizon parametrized by the intervals of $(0, \infty)$, where the Killing automorphism group $\alpha$ acts covariantly by dilations, cf. equation (2.4). We denote by $\mathfrak{C}(0, \infty)$ the $C^{*}$-algebra generated by all $\mathcal{C}(a, b), b>a>0$.

We shall say that a one-parameter unitary group $T$ implements translations on the horizon if equations (2.3) and (1.13) are satisfied.

Corollary 2.7. Assume that $\mathcal{C}(0, a)$ is $\frac{\beta}{2}$-bounded w.r.t. $K$ and $\xi$ for some $\beta \geq \beta_{0}$ and $a>0$, where $\beta_{0}=\frac{2 \pi}{\kappa}$ is the inverse of the Hawking temperature. The following are equivalent:

(i) $\left.\varphi\right|_{\mathfrak{C}(0, \infty)}$ is a KMS state at Hawking inverse temperature $\beta_{0}$.

(ii) There exists a one-parameter unitary group $T$ implementing translations on the horizon.

In this case the generator of $T$ is positive and the net extends to a conformal net on the line. 
Proof. $(i) \Rightarrow(i i)$. The inclusion $\mathcal{R}_{1} \subset \mathcal{R}_{0}$ is half-sisd modular, therefore the translations (with positive generator) can be constructed as in 442, cf. also Proposition4A.2 in [19].

(ii) $\Rightarrow(i)$. First we extend the net $\mathcal{C}$ to all intervals in $\mathbb{R}$ setting $\mathcal{C}(a, b)=$ $T(a)^{*} \mathcal{C}(0, b-a) T(a), a<0$. Clearly $T$ and $U$ act as translations and dilations on the net, therefore Proposition 2.5 applies, hence the generator of $T$ is positive. Conformal invariance then follows by [43].

\section{Conclusion}

We have seen that states with the $\beta$-boundedness conditions are indeed thermal equilibrium states in certain Quantum Field Theory contexts. In general the $\beta$ boundedness condition selects particular non-equilibrium steady states, whose meaning is not completely clear.

One may be tempted to use the $\beta$-boundedness condition to define the "local temperature" of an observable $X$ as the inverse of $\sup \left\{\beta>0: X \Omega \in D\left(e^{-\frac{\beta}{2} K}\right)\right\}$ with the notations in the text, cf. [11].

This suggests the following physical interpretation. If a thermodynamical system $\Sigma$ sits in the background of the black hole, interacts with heat reservoirs at temperature less than the Hawking temperature, then the black hole is a predominant heat bath for $\Sigma$ and the state is a thermal equilibrium state at the black hole background temperature. In particular, the Hawking temperature is minimal and one cannot cool the system $\Sigma$ down by letting it interact with an infinite reservoir at lower temperature. However, the above discussion relies on the assumption that only $\beta$-holomorphic states enter in the game.

The validity of the above picture relies on a clarification of the role of the $\beta$-holomorphic states, namely how large is their class and how close they are to equilibrium states.

Acknowledgments. We would like to thank C. D' Antoni for conversations.

\section{References}

[1] H. Araki, G.L. Sewell, KMS condition and local thermodynamic stability of quantum lattice systems, Commun. Math. Phys. 52, 103-109 (1977).

[2] J.D. Bekenstein, Generalized second law of thermodynamics in black hole physics, Phys. Rev. D 9 (1974), 3292-3300

[3] P. Bertozzini, R. Conti, R. Longo, Covariant sectors and positivity of the energy, Commun. Math. Phys. 141, 471-492 (1998).

[4] J.J. Bisognano, E.H. Wichmann, On the duality condition for quantum fields, J. Math. Phys. 17, 303 (1976).

[5] H.-J. Borchers, The CPT-theorem in two-dimensional theories of local observables, Commun. Math. Phys. 143, 315 (1992). 
[6] H.-J. Borchers, Energy and momentum as observables in Quantum Field Theory, Commun. Math. Phys. 2, 49 (1966)

[7] O. Bratteli, D. Robinson, "Operator algebras and quantum statistical mechanics. 2. Equilibrium states. Models in quantum statistical mechanics", 2nd ed., Springer, Berlin, 1997.

[8] D. Buchholz, C. D'Antoni, R. Longo, Nuclear maps and modular structures I. General properties, J. Funct. Anal. 88 (1990), 223-250.

[9] D. Buchholz, C. D'Antoni, R. Longo, Nuclear maps and modular structures II. Applications to quantum field theory, Commun. Math. Phys. 129 (1990), 115-138.

[10] D. Buchholz, E.H. Wichmann, Causal independence and the energy-level density of states in local quantum field theory, Commun. Math. Phys. 106 (1986), 321-344.

[11] C. D'Antoni, L. Zsido, in preparation.

[12] S. Doplicher, R. Longo, Standard and split inclusions of von Neumann algebras, Invent. Math. 73 (1984), 493-536.

[13] F. Fidaleo, Operator space structures and the split property, J. Operator Theory 31 (1994), 207-218.

[14] F. Figliolini, D. Guido, On the type of second quantization factors, J. Operator Theory, 31 (1994), 229-252

[15] M. Florig, On Borchers' theorem, Lett. Math. Phys. 46 (1998), 289-293.

[16] D. Guido, R. Longo, An algebraic spin and statistics theorem, Commun. Math. Phys. 172 (1995), 517-533.

[17] D. Guido, R. Longo, The conformal spin and statistics theorem Commun. Math. Phys. 181 (1996), 11-35.

[18] D. Guido, R. Longo, H.-W. Wiesbrock, Extension of conformal nets and superselection structures, Commun. Math. Phys. 192, 217-244 (1998).

[19] D. Guido, R. Longo, J.E. Roberts, R. Verch, Charged sectors, spin and statistics in quantum field theory on curved space-times, Rev. Math. Phys. (to appear), math-ph/9906019.

[20] R. Haag, "Local quantum physics", 2nd ed., Springer, Berlin, Heidelberg, New York, 1996

[21] R. Haag, N.M. Hugenoltz, M. Winnik, On the equilibrium states in quantum statistical mechanics, Commun. Math. Phys. 5 (1967), 215-236.

[22] R. Haag, J.A. Swieca, When does a quantum field theory describe particles? Commun. Math. Phys. 1 (1965), 308-320. 
[23] U. Haagerup, Solution of the similarity problem for for cyclic representations of $C^{*}$-algebras, Annals of Math. 118 (1983), 215-240.

[24] S.W. Hawking, Particle creation by black holes, Commun. Math. Phys. 43, 199 (1975).

[25] B. S. Kay, R. M. Wald, Theorems on the uniqueness and thermal properties of stationary, nonsingular, quasifree states on space-times with a bifurcate killing horizon, Phys. Rept. 207 (1991), 49-136.

[26] R. Longo, Algebraic and modular structure of von Neumann algebras of physics, Proc. Symp. Pure Math. 38, (1982), Part 2, 551.

[27] R. Longo, An analogue of the Kac-Wakimoto formula and black hole conditional entropy, Commun. Math. Phys. 186 (1997), 451-479.

[28] R. Longo, Notes for a quantum index theorem, preprint, March 2000.

[29] G.K. Pedersen, "C*-algebras and their automorphism group", Academic Press, London, 1979.

[30] G. Pisier, "Similarity problems and completely bounded maps", Lecture Notes in Mathematics 1618, Springer-Verlag, Berlin, 1996.

[31] G. Pisier, Grothendieck's theorem for noncommutative $C^{*}$-algebras with an appendix to Grothendieck's constants, J. Funct. Anal. 29 (1978), 397-415.

[32] W. Pusz, S.L. Woronowicz, Passive states and KMS states for general quantum systems, Commun. Math. Phys. 58 (1978), 273-290.

[33] G. Roepstorff, Correlation inequalities in quantum statistical mechanics and their applications to Kondo problem, Commun. Math. Phys. 46 (1976), 253-262.

[34] D. Ruelle, Natural nonequilibrium states in quantum statistical mechanics, J. Statist. Phys. 98 (2000), 57-75.

[35] B. Schroer, H.W. Wiesbrock, Modular theory and geometry, Rev. Math. Phys. 12 (2000), 139-158.

[36] G.L. Sewell, Quantum fields on manifolds: PCT and gravitationally induced thermal states, Ann. Phys. (N.Y.) 141, 201 (1982)

[37] S. Stratila, L. Zsidó, "Lectures on von Neumann algebras", Abacus Press, Tunbridge Wells, Kent (1979).

[38] M. Takesaki, "Theory of Operator Algebras", vol. II, in preparation.

[39] W.G. Unruh, Notes on black hole evaporation, Phys. Rev. D 14 870, (1976).

[40] R.M. Wald, "Quantum field theory in curved spacetime and black hole thermodynamics", University of Chicago Press, 1994 
[41] H.-W. Wiesbrock, A comment on a recent work of Borchers, Lett. Math. Phys. 25 (1992), 157-159.

[42] H.-W. Wiesbrock, Half-sided modular inclusions of von Neumann algebras, Commun. Math. Phys. 157, 83 (1993)

[43] H.-W. Wiesbrock, Conformal quantum field theory and half-sided modular inclusions of von Neumann algebras, Commun. Math. Phys. 158, 537 (1993) 